\title{
Computing valuation adjustments for counterparty credit risk using a modified supervisory approach
}

\author{
Patrick Büchel ${ }^{1} \cdot$ Michael Kratochwil ${ }^{2,3}$ (1) Daniel Rösch ${ }^{2}$ \\ Published online: 14 January 2020 \\ (c) The Author(s) 2020
}

\begin{abstract}
Considering counterparty credit risk (CCR) for derivatives using valuation adjustments (CVA) is a fundamental and challenging task for entities involved in derivative trading activities. Particularly calculating the expected exposure is time consuming and complex. This paper suggests a fast and simple semi-analytical approach for exposure calculation, which is a modified version of the new regulatory standardized approach (SA-CCR). Hence, it conforms with supervisory rules and IFRS 13. We show that our approach is applicable to multiple asset classes and derivative products, and to single transactions as well as netting sets.
\end{abstract}

Keywords Counterparty credit risk - Credit valuation adjustments (CVA) - Credit exposure $\cdot$ Standardized approach for measuring counterparty credit risk exposures (SA-CCR)

JEL Classification G21 - G32

The authors would like to thank the participants of the Quantitative Methods in Finance (QMF)
Conference 2018 in Sydney for helpful comments. We would also like to thank two anonymous referees
for comments which greatly helped us improving the paper.

$\bowtie \quad$ Michael Kratochwil

michael.kratochwil@ur.de

Patrick Büchel

patrick.buechel@commerzbank.com

Daniel Rösch

daniel.roesch@ur.de

1 Commerzbank AG, Mainzer Landstraße 157, 60327 Frankfurt am Main, Germany

2 Chair of Statistics and Risk Management, Universität Regensburg, Universitätsstraße 31, 93040

Regensburg, Germany

3 Dr. Nagler \& Company GmbH, Maximilianstraße 47, 80538 Munich, Germany 


\section{Introduction}

The financial crisis and its aftermath have revealed the importance of counterparty credit risk (CCR) in over-the-counter (OTC) derivative transactions. Today, the consideration of CCR is market standard and the calculation of credit valuation adjustments (CVA) has evolved to be a fundamental task for entities involved in derivatives trading due to several reasons. Firstly, market participants need to consider CCR when pricing derivatives. Secondly, international financial reporting standards (IFRS 13) require all entities involved in derivative transactions to consider CCR in the accounting fair value. ${ }^{1}$ Thirdly, financial institutions are expected to calculate minimum capital requirements for CVA risk under Basel III, which implies the calculation of CVA as well as CVA sensitivities. The most time-consuming and complex part of xVA calculation is the determination of the expected exposure. Given the lack of clear methodological guidance in IFRS 13, a wide range of methods has been developed by regulators, financial institutions and scientists alike. As many market participants may not be able to apply highly complex and sophisticated methods, there is a need for simpler semi-analytical and parametric approaches. Most existing approaches are either too simplistic to be robust, only applicable on transaction level or suitable for a small range of products. Hence, most of these methods are not applicable to multidimensional netting sets.

Our paper provides the following contributions. Firstly, we develop a fast and simple semi-analytical method for exposure calculation, which is a modified version of the new supervisory standardized approach for measuring counterparty credit risk exposures (SA-CCR). We derive the necessary adjustments to the regulatory SA-CCR in order to ensure consistency with IFRS 13. The approach has a flexible structure and is able to capture risk mitigating effects from margining and collateralization. Secondly, we show that our approach is applicable to multiple asset classes and on a singletransaction as well as a netting set level. To ensure the usability of our approach, we compare our results with an advanced model approach for an illustrative set of interest rate and foreign exchange derivatives.

We find that our modified SA-CCR approach is able to produce expected exposure profiles capturing the main exposure dynamics of interest rate and foreign exchange positions. Hence, we are able to mirror exposure profiles generated by advanced methods, which might serve as input for CVA calculation. As we maintain the key building blocks and methodological assumptions of the supervisory SA-CCR, we offer a flexible and consistent approach to calibration based on market-implied volatilities, yet simple enough to be adopted by smaller institutions with limited personal resources.

The remainder of the paper is structured as follows. Section 2 provides an overview and categorization of existing methods for exposure quantification. In Sect. 3 we derive the necessary adjustments to the SA-CCR based on central model foundations. The calibration of the modified SA-CCR is lined out in Sect. 4. The methodology and results of the empirical analysis are presented in Sect. 5. Section 6 concludes this paper.

\footnotetext{
1 IFRS 13 also requires the inclusion of an entity's own credit risk in the fair value measurement via the calculation of Debit Valuation Adjustments (DVA). In this paper we focus solely on the calculation of CVA based on the expected positive exposure. It is possible to adapt our approach for calculation of further xVAs.
} 


\section{Methods for exposure quantification}

Calculation of $x$ VAs requires the quantification of the expected exposure at time $t$. The lack of clear guidance from accountants and supervisors as well as the need for complex and simpler methods have led to the development of a wide range of approaches. According to Gregory (2015), these methods can be divided into advanced, parametric and semi-analytical approaches. Using advanced approaches is the most sophisticated way to quantify CCR exposures, and there is plenty of academic literature on their application (see Pykhtin and Zhu 2007; Pricso and Rosen 2005; Picoult 2004; Canabarro and Duffie 2003; Picoult 2004). An advanced approach provides the most realistic risk assessment, but requires in-depth quantitative knowledge, a multitude of input data and a powerful infrastructure. Especially the simulation of potential market scenarios and the valuation of transactions for each scenario and viewpoint are complex and laborious tasks. Developing and maintaining an advanced model is complex and associated with high costs. While advanced approaches are usually applied in larger financial institutions, small and medium-sized market participants often do not have the capabilities to operate a complex exposure simulation model. Thompson and Dahinden (2013) find that even banks applying advanced models are often unable to cover all asset classes and products within these models. Therefore, we are certainly justified in saying that there is a need for alternative, less sophisticated approaches. To avoid an operational burdensome simulation model, various semi-analytical methods have been developed. These approaches are based on assumptions with respect to the development of risk factors driving the market value of a product or netting set. One prominent example for semi-analytical methods is the swaption approach introduced by Sorensen and Bollier (1994). They measure the exposure of an interest rate swap by valuing a series of swaptions, which a party would theoretically enter into in case of the counterparty's default. There are several other semi-analytical methods for interest rate swaps and other derivative products (such as Leung and Kwok 2005 for credit default swaps). In the past years, there has been a lot of work on the development and enhancement of reduced-form and structural models for CVA calculation (e.g. Kao 2016; Hull and White 2012; Cherubini 2013). While semi-analytical methods are considered the best choice for modelling CCR exposure on transaction level, their application is limited. Semi-analytical methods are generally suited for a limited number of products and designed for a specific asset class. Hence, it is difficult to apply these methods for products with multiple underlying risk factors (e.g. cross-currencyswaps) and multi-dimensional netting sets. In general, CCR exposures are calculated on netting set/counterparty level and require an aggregation of product-specific exposure profiles, which is something most of these semi-analytical methods are not able to provide. Most semi-analytical approaches ignore diversification and effects from collateralization, netting and margining. Even extensions are only able to recognize these effects in a very limited way. For example, Brigo and Masetti (2005) develop an analytical approach for interest rate portfolios in a single currency.

Parametric approaches are considered to be the most simplistic way of quantifying CCR exposures. They provide an approximation based on a limited number of simple parameters. Most parametric approaches calculate the exposure as the sum of current exposure $(C E)$ and an add-on for potential future exposure $(P F E)$. By 
calibrating the aforementioned simple parameters to more complex methods, the outcome of parametric approaches is aligned with the results from more sophisticated models. Especially regulatory standardized approaches are based on the idea of simplification and calibration. When calculating the exposure at default $(E A D)$ for the assessment of minimum regulatory capital requirements, banks currently have the option to choose between using an advanced Internal Model Method (IMM) or one of two standardized approaches (Standardized Method (SM), Current Exposure Method (CEM)). ${ }^{2}$ According to EBA (2016) the Current Exposure Method (CEM) is the most widespread approach for calculating CCR exposures in the European banking sector for regulatory purposes. This method was introduced by the Basel Committee on Banking Supervision (BCBS) (1996) and is still valid after it was adjusted in the course of Basel II (2005). A majority of financial institutions uses methods based on the CEM for accounting and pricing purposes. These approaches are often referred to as "mark-to-market plus add-on" methods. In the past, especially the CEM was criticized for several reasons. From the perspective of BCBS (2014c) the main issues are (1) the lack of risk sensitivity, (2) the outdated calibration of risk weights, (3) the missing ability to recognize credit risk mitigation techniques (in particular margining) as well as (4) a too simplistic attempt to capture netting effects. ${ }^{3}$

Driven by this criticism, the financial crisis and the increasing importance of bilateral margining in OTC derivatives markets, ${ }^{4}$ a new regulatory standardized approach was developed by the BCBS (2014c). The SA-CCR will replace the existing standardized approaches (SM and CEM). With the development of the SA-CCR, the BCBS was striving for a holistic approach applicable to a variety of derivative products. Furthermore, the SA-CCR was intended to overcome the weaknesses of existing approaches while keeping complexity on a reasonable level.

The SA-CCR can be classified as a semi-analytic method. It uses a rule based calculation scheme and simple parameters. Nevertheless, the derivation of the approach is based on detailed assumptions with respect to the distribution of market values and model based aggregation algorithms. The SA-CCR has several major advantages compared to its predecessors. First, the SA-CCR is able to distinguish between margined and unmargined netting sets. Effects of margining are considered in the current and potential future exposure component. This is an important feature in light of the rising importance of bilateral margining and central clearing. As stated in BCBS (2014c) the SA-CCR is also able to cope with complex situations (e.g. several netting sets are covered by one margin agreement). Second, the SA-CCR applies a more sophisticated approach to netting and diversification. This adds additional complexity, but should lead to higher risk sensitivity in the approximation of exposures (BCBS 2014c). The

\footnotetext{
2 Institutions with very limited trading business have the possibility to use an even simpler method, the Original Exposure Method (OEM), for the purpose of calculating minimum regulatory capital requirements.

3 For a comprehensive discussion of critique of CEM, please refer to Fleck and Schmidt (2005) and Pykhtin (2014).

4 As a result of the global financial crises, regulators all over the world set regulations for reducing risk in the financial industry, especially in the OTC market, and to protect counterparties from the risk of a potential default of the other counterparty. These are the obligation to clear certain derivative products as well as the obligation to reduce the risk of non-cleared OTC derivative contracts by exchanging collateral in form of initial and variation margin (BCBS, IOSCO 2015; ESAs 2016).
} 
structure of the calculation of potential future exposure is flexible and allows to add or delete elements where necessary. ${ }^{5}$ Third, the SA-CCR takes over-collateralization, moneyness of transactions and the netting set into account. As excess collateral and transactions with negative values guard against rising exposures, this should lead to more realistic results. Overall, the SA-CCR is more complex compared to the popular CEM, but financial institutions might be able to leverage on the improved risk sensitivity and flexibility. The SA-CCR provides a consistent exposure calculation framework for all asset classes, while accounting for specific aspects of different financial products (such as equity options, swaptions, etc.). Considering these facts alongside the aforementioned improvements and the transparency with respect to its model foundations, the application of SA-CCR for CVA pricing and accounting purposes is an interesting option for all kinds of entities involved in derivatives trading.

According to Marquart (2016) the application of regulatory approaches (foremost CEM) is considered best practice when calculating exposures for CVA pricing. She analysed the impact on accounting CVA when switching from CEM to SA-CCR, under the assumption of using a simple CVA formula $C V A=P D \cdot L G D \cdot E A D$, where $E A D$ is defined as the Effective Expected Positive Exposure ( $E E P E$ ) resulting from SA-CCR, or CEM respectively. The application of the supervisory SA-CCR for CVA pricing compasses several issues. First, the SA-CCR aims for an approximation of the exposure at default ( $E A D)$ under the Internal Model Method (IMM). Under IMM, the $E A D$ is defined as the product of the Effective Expected Positive Exposure (EEPE) and a factor $(\alpha=1.4)$, which is used to convert the $E E P E$ into a loan equivalent exposure. ${ }^{6}$ For CVA pricing, a time dependent expected exposure profile $E E(t)$ is required. Hence, the target measure of the supervisory SA-CCR is not appropriate. Second, the SA-CCR is calculated for a risk horizon of up to one year for unmargined netting sets. For the purpose of CVA pricing, an exposure profile for the life-time of a netting set is required. Using the $E E P E$ or $E A D$ as a scalar when calculating CVA would ignore the time dependency of exposure. Third, the SA-CCR is calibrated to a period of stress. This means resulting exposures are calculated under the real-world measure. According to IFRS 13, the calculation of CVA needs to be conform to the expectations of market participants. This requires a calibration under the risk-neutral measure. Additionally, the SA-CCR contains a set of conservative elements which should not be applied when calculating exposure for CVA pricing. In conclusion, we find that the SA-CCR in its supervisory form does not conform to IFRS 13. Hence, modifications to the regulatory SA-CCR are required to deploy the approach for CVA pricing and accounting purposes.

\section{Derivation of the modified SA-CCR}

This paper aims to define modifications to the supervisory SA-CCR to derive an approach for the calculation of expected exposure profiles. As stated above, the adjust-

\footnotetext{
5 There are discussions to give national competent authorities the option to adjust the add-on structure for institutions with complex commodity trading activities.

6 For information on the calibration and theoretical background of $\alpha$, please refer to ISDA, TBMA, LIBA (2003), Lynch (2014) and Gregory (2015).
} 
ments are necessary in order to calculate exposure values suitable for CVA calculations. While adjusting the SA-CCR, we aim to retain the basic structure and main building blocks. This allows the application of a consistent approach across asset classes and enables financial institutions to leverage on future implementations of the supervisory SA-CCR. The following presentation of the SA-CCR methodology and the derivation of its adjustments is based on the content and structure of BCBS (2014b).

The calculation of CVA requires an expected exposure profile as the main input. In the absence of collateral, the expected exposure of a netting set $(k)$ is defined as the expected positive value of the netting set's market value $\left(V_{k}\right)$ at a future point in time $(t)$ :

$$
E E_{k}(t)=\mathbb{E}^{Q}\left[\max \left(V_{k}(t), 0\right)\right]
$$

In its supervisory form, the target measure of the SA-CCR is a conservative Effective Expected Positive Exposure ( $E$ E P E ) on netting set level under the real-world measure (calibrated to historic stressed volatilities). Hence, the main adjustment when deriving our approach is the change of target measure to an $E E_{k}(t)$ under the risk-neutral measure. To retain the general structure of the SA-CCR, we define $E E_{k}(t)$ as the combination of replacement costs $\left(R C_{k}(t)\right)$ and potential future exposure $\left(P F E_{k}(t)\right)$ :

$$
E E_{k}(t)=R C_{k}(t)+P F E_{k}(t)
$$

Please note that both components of the modified SA-CCR are a function of time $(t)$. Following our approach, $R C_{k}(t)$ captures the deterministic component, while potential future exposure quantifies the stochastic component of $E E_{k}(t)$. In the following sections, we derive the modified formulas for calculation of these components on netting set level. Finally, we transfer our results to the SA-CCR specific parameters for exposure calculation.

\subsection{Replacement costs}

For the derivation of the replacement costs formula, we first introduce the following assumptions. (A1) A transaction's market value follows a driftless brownian motion. For the formulation of replacement costs, we set the volatility to zero. (A2) We assume no cash flows between $\left(t_{0}, t\right)$. (A3) Furthermore, the transaction's netting set is unmargined and therefore not supported by a margin process. ${ }^{7}$ These assumptions are used implicitly by BCBS (2014b) for the derivation of RC for unmargined netting sets.

Under these assumptions, the future market value of a transaction $\left(V_{i}\right)$ at a specific point in time $(t)$ is defined as:

$$
V_{i}(t)=V_{i}\left(t_{0}\right)+\sigma_{i}(t) \cdot \sqrt{t} \cdot X_{i}
$$

\footnotetext{
7 A netting set is considered to be unmargined if there is no exchange of variation margin $(V M)$. Nevertheless, other types of collateral (such as initial margin $(I M)$ ) might be present.
} 
where $V_{i}\left(t_{0}\right)$ represents today's market value and $X_{i}$ is a standard normal random variable $\left(X_{i} \sim N(0,1)\right) . \sigma_{i}(t)$ represents the volatility of the transaction's market value at time $t$. Applying assumption A1, the expected future market value is equal to today's market value as the second term of Eq. (3) becomes zero. As stated above, $R C(t)$ should capture the deterministic movements of a transaction's market value. In particular, interest rate and credit default swaps involve regular payments resulting in a change of the transaction's market value over time. In order to cover these deterministic effects, we relax assumption A2. Given assumption A1, the future market value of a transaction is deterministic and can be calculated based on the transaction's future cash flows. This may generally be written as:

$$
\hat{V}_{i}(t)=\sum_{j=t}^{T} C F_{R E C}\left(t_{j}\right) \cdot D F\left(t, t_{j}\right)-\sum_{j=t}^{T} C F_{P A Y}\left(t_{j}\right) \cdot D F\left(t, t_{j}\right)
$$

where $C F_{R E C}\left(t_{j}\right)$ is the cash flow received at time $t_{j}, C F_{P A Y}\left(t_{j}\right)$ equals the cash flow paid at time $t_{j}$ and $D F\left(t, t_{j}\right)$ represents the discount factor from time $t_{j}$ to time $t$.

For more complex derivatives or in case no information regarding future cash flows is available, we introduce a time-dependent and product specific scaling factor $\left(s_{i}\right)$ to provide an approximation of the future market value of a transaction $\left(\hat{V}_{i}(t)\right)$.

$$
\hat{V}_{i}(t)=V_{i}\left(t_{0}\right) \cdot s_{i}(t)
$$

For interest rate or credit default swaps, this scaling factor might be based on a simplified duration measure for the respective product:

$$
s_{i}(t)=\frac{D_{i}(t)}{D_{i}\left(t_{0}\right)} \cdot 1_{\left\{M_{i} \geq t\right\}}
$$

where $1_{\left\{M_{i} \geq t\right\}}$ is an indicator variable which has the value of 1 if the transaction has not expired at $t$ (i.e., maturity $M_{i}$ is greater or equal than $t$ ). The duration measure $D_{i}(t)$ is defined as: ${ }^{8}$

$$
D_{i}(t)=\frac{\exp \left(-r \cdot \max \left(S_{i}, t\right)\right)-\exp \left(-r \cdot E_{i}\right)}{r}
$$

where $S_{i}$ is the start date of the transaction and $E_{i}$ its end date. $r$ is defined as the current interest rate level. For simple products in other asset classes, $s_{i}^{(a)}$ could be represented by the indicator variable. Nevertheless, our approach offers the flexibility to define a transaction specific scaling factor for all kinds of (exotic) products. This allows a recognition of deterministic developments of the transaction's market value in a flexible and consistent setting.

Within a legally enforceable netting set $(k)$, the offsetting between transactions with positive and negative market values $\left(\hat{V}_{i}(t)\right)$ is allowed. Hence, a netting set's market value at time $t$ is defined as:

\footnotetext{
${ }^{8}$ For the derivation of $D_{i}(t)$ please refer to "Appendix A.3.3".
} 


$$
\hat{V}_{k}(t)=\sum_{i \in k} \hat{V}_{i}(t)
$$

As stated above, replacement costs do not involve stochastic elements. Thus, the expectation of the future market value is solely driven by deterministic movements and hence represented by $\hat{V}_{k}(t)$. This leads to the following formulation of replacement costs for unmargined and uncollateralized netting sets:

$$
R C_{k}(t)=\mathbb{E}^{Q}\left[\max \left(V_{k}(t), 0\right)\right]=\max \left(\hat{V}_{k}(t), 0\right)
$$

In the presence of collateral, the market value of the netting set is reduced by the cash-equivalent value of net collateral received $\left(C_{C E}(t)\right)$. Under assumption $\mathbf{A 3}$, all collateral posted or received has the form of independent collateral. Given the lack of a margin process, no adjustment to the notional amount of collateral posted/received is required. The time dependency of the collateral value is limited to the volatility of the collateral value itself. In accordance with the supervisory SA-CCR, we calculate cashequivalent values of collateral $\left(C_{C E}(t)\right)$ using collateral haircuts. There are two main adjustments to the supervisory approach. Firstly, we do not use a fixed time horizon, but calculate the cash-equivalent value for specific points in time $(t)$. Secondly, we do not apply regulatory prescribed haircuts, but values based on institutions' own volatility estimates. Given these adjustments, $C_{C E}(t)$ is defined as:

$$
C_{C E}(t)=\sum_{c \in k} V_{c}^{r e c}\left(t_{0}\right) \cdot\left(1-h_{c}(t)\right)-\sum_{c \in k} V_{c}^{\text {post }, \text { unseg }}\left(t_{0}\right) \cdot\left(1+h_{c}(t)\right)
$$

where $V_{c}$ equals the market value of a received $\left(V_{c}^{r e c}\left(t_{0}\right)\right)$ or unsegregated posted

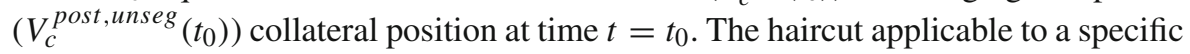
collateral position is represented by $h_{c}(t)$. Please note that segregated posted collateral is not relevant for the calculation of replacement costs, as it is placed in a bankruptcy remote account and will therefore not increase exposure to the relevant counterparty. Including collateral positions in the calculation of replacements costs for unmargined netting sets leads to:

$$
R C_{k}(t)=\max \left(\hat{V}_{k}(t)-C_{C E}(t), 0\right)
$$

In order to derive a formulation for margined netting sets, we need to relax assumption A3. Within the modified SA-CCR, we introduce the possibility to model collateral dynamics directly. We calculate the future expected market value of each transaction at each point in time $t$ based on known cash flows using Eq. (4) or by applying a scaling factor (see Eq. (5)). Hence, we know the expected future market value of the netting set $\left(\hat{V}_{k}(t)\right)$ at each $t$. Based on this information, we are able to derive an expected collateral path including the consideration of margin parameters like threshold $(T H)$ and Minimum Transfer Amount $(M T A)$. In case of $(T H \neq 0)$ collateral is only exchanged, when the threshold is exceeded. This implies that the incremental amount above the threshold is exchanged in form of collateral. We define the amount above 
the threshold as the collateral demand $\left(\hat{C_{D}}(t)\right)$. If $(M T A \neq 0)$, collateral is only exchanged, when the absolute difference between the current collateral position $(\hat{C}(t))$ and the collateral demand exceeds the $M T A$. Under the assumption of symmetric $M T A$ and $T H$, absence of rounding, daily margining and instantaneous processing of collateral exchange, the expected collateral position at $t_{j}$ is defined as: ${ }^{9}$

$$
\begin{aligned}
\hat{C}\left(t_{j}\right)= & \hat{C}\left(t_{j-1}\right)+\max \left(\max \left(\hat{C}_{D}\left(t_{j}\right)-\hat{C}\left(t_{j-1}\right), 0\right)-M T A, 0\right) \\
& +\min \left(\min \left(\hat{C}_{D}\left(t_{j}\right)-\hat{C}\left(t_{j-1}\right), 0\right)+M T A, 0\right)
\end{aligned}
$$

where the collateral demand $\left(\hat{C_{D}}(t)\right)$ is defined as:

$$
\begin{aligned}
\hat{C_{D}}\left(t_{j}\right)= & \max \left(\max \left(\hat{V}\left(t_{j}\right), 0\right)-T H, 0\right) \\
& +\min \left(\min \left(\hat{V}\left(t_{j}\right), 0\right)+T H, 0\right)
\end{aligned}
$$

Based on this definition, the replacement costs for a margined netting set at $t$ are defined as: ${ }^{10}$

$$
R C_{k}^{\text {margin }}(t)=\max \left(\hat{V}_{k}(t)-\hat{C}(t)+N I C A, 0\right)
$$

where NIC A represents the Net Independent Collateral Amount defined as:

$$
N I C A=I M_{r e c}-I M_{p o s t}^{u n s e g}
$$

In addition to modelling collateral dynamics directly, we introduce an optional (alternative), more simplistic approximation for the recognition of collateral in margined netting sets. This conservative approximation follows the methodology described in BCBS (2014b). We assume that the latest exchange of variation margin is not known at time $t$. Hence, we estimate $R C(t)$ of margined netting sets as the maximum of replacement costs of an equivalent unmargined netting set, the highest exposure amount which would not trigger a margin call and zero. In general, a margin call is triggered if the uncollateralized market value is equal to the sum of $T H$ and $M T A$. This amount is reduced by the net independent collateral amount $(N I C A) .{ }^{11}$

Under a margin agreement, changes in the netting set's market value will lead to changes in the amount of variation margin posted or received. Therefore, we introduce a time-dependent adjustment for variation margin $(V M)$ based on the change of the market value of the netting set. Based on this adjustment, we arrive at the following

\footnotetext{
9 Please note that our approach offers the possibility to integrate additional collateral parameters, such as independent amounts, rounding or other re-margining periods.

10 Please note that the application of haircuts is also required for margined netting sets. In case of a margined netting set, the risk horizon for the application of haircuts is set to the MPOR.

11 In this case NICA has to include differential of the independent amounts used as parameters within the calculation of variation margin amounts.
} 
approximation of replacement costs for margined netting sets:

$$
R C_{k}^{\text {margin }}(t) \approx \max \left(\hat{V}_{k}(t)-\hat{C}_{C E}(t), T H+M T A-N I C A, 0\right)
$$

where $\hat{C}_{C E}(t)$ is defined as:

$$
\hat{C}_{C E}(t)=\left(\sum_{c \in k} V_{c}^{V M}\left(t_{0}\right) \cdot\left(1 \pm h_{c}(M P O R)\right)\right) \cdot \frac{\hat{V}_{k}(t)}{V_{k}\left(t_{0}\right)}+N I C A
$$

where $V_{c}^{V M}\left(t_{0}\right)$ is defined as today's market value of a variation margin collateral position. ${ }^{12}$ In general we cap the expected exposure of a margined netting set at the expected exposure of an equivalent netting set without any form of margin agreement. This is equal to the assumption that a netting set is treated as unmargined as long as no collateral is exchanged (e.g. the sum of MTA and TH is not exceeded).. This procedure is required to avoid overly conservative results due to high thresholds and minimum transfer amounts.

\subsection{Potential future exposure}

In line with BCBS (2014b) we define the potential future exposure $(P F E)$ as the product of a multiplier $\left(m_{k}\right)$ and an aggregated add-on $\left(A d d O n_{k}\right)$ for each netting set $(k)$ :

$$
P F E_{k}(t)=m_{k}(t) \cdot A d d O n_{k}(t)
$$

where $m_{k}(t)$ is a function of $\hat{C}_{C E}(t), \hat{V}_{k}(t)$ as well as the calculated aggregated add-on $\left(A d d O n_{k}(t)\right)$ of the respective netting set $(k)$. In our approach, the aggregated addon represents an analytical approximation of $E E_{k}(t)$ on netting set level, assuming a market value of zero and the absence of collateral. The multiplier is introduced to account for market value and collateral amounts different from zero. The regulatory SA-CCR approach reflects the benefit of excess collateral and negative market values, as only these are mitigants against potential future exposure. Please note that the multiplier as well as the aggregated add-on are a function of time $(t)$. In the subsequent paragraphs we provide the derivation of add-ons as well as the multiplier formula.

\subsubsection{Add-ons for unmargined netting sets}

The netting set level add-on for unmargined netting sets represents an estimate of the expected exposure $(E E)$ at time $t$. The assumptions of the regulatory SA-CCR presented in BCBS (2014b) are maintained in order to build a consistent and integrated framework. Hence, our approach is based on the following main assumptions:

\footnotetext{
12 Please note that a change in sign of $\hat{V}_{k}(t)$ will also lead to a change in sign of variation margin. In case of posted VM, segregated collateral needs to be eliminated from the calculation of $\hat{C}_{C E}(t)$. Hence, assumptions on the properties of potentially posted and received variation margin are required.
} 
- A01: The market value of all transactions is zero $\left(V_{i}(t)=0\right)$. This assumption implies that the market value of the netting set is zero $\left(V_{k}(t)=0\right)$.

- AO2: There is neither received nor posted collateral $\left(C_{C E}(t)=0\right)$.

- A03: There are no cash-flows within the time period $\left(t_{0}, t\right)$

- AO4: The evolution of each transaction's market value follows an arithmetic brownian motion with zero drift.

Under these assumptions, the expected exposure of a netting set at time $t$ is defined as: ${ }^{13}$

$$
E E_{k}(t)=\mathbb{E}^{Q}\left[\max \left(V_{k}(t), 0\right)\right]=\mathbb{E}^{Q}\left[\max \left(\sigma_{k}(t) \cdot \sqrt{t} \cdot Y, 0\right)\right]
$$

with $\sigma_{k}(t)$ representing the annualized volatility of the netting set's market value at $t$. As $Y$ is a standard normal variable, we can calculate $E E_{k}(t)$ analytically. Hence, the expected exposure solves for: ${ }^{14}$

$$
E E_{k}(t)=\sigma_{k}(t) \cdot \sqrt{t} \cdot \phi(0)
$$

where $\phi(0)$ is defined as the standard normal probability density: $\phi(0)=1 / \sqrt{2 \pi}$. According to BCBS (2014b) and in line with the above foundations, we are able to restate this equation at trade level in order to calculate an expected exposure at trade level $E E_{i}(t)$.

$$
A d d O n_{i}(t)=E E_{i}(t)=\sigma_{i}(t) \cdot \sqrt{t} \cdot \phi(0)
$$

Please note that contrary to BCBS (2014b) the volatility of the market value on trade level $\left(\sigma_{i}(t)\right)$ is a function of $t$ as we estimate the volatility of each transaction's market value as a function of $t$. Nevertheless, we are generally able to use the same structure and aggregation methodology for the calculation of add-ons as proposed by BCBS $(2014 b){ }^{15}$

\subsubsection{Add-ons for margined netting sets}

The add-on for margined netting sets aims to estimate the expected increase of exposure between time of default $(\tau=t)$ and the final close-out of positions $(t+M P O R)$. Given assumptions AO1, AO2 and AO3 and in accordance with the argumentation of BCBS (2014b), the calculation of this amount on netting set level can be reduced to:

$$
\operatorname{AddOn}_{k}^{\text {margin }}(t)=E E_{k}^{\text {margin }}(t)=\sigma_{k}(t) \cdot \phi(0) \cdot \sqrt{M P O R}
$$

\footnotetext{
13 For detailed derivation of Eq. (19), please refer to "Appendix A.1".

14 For the respective derivation of the analytical formulation of $E E_{k}(t)$, please refer to "Appendix A.1".

15 The validity of this assumption under the new target measure $E E(t)$ is proven in "Appendix A.2".
} 
For a netting set with only one trade, we can restate formula (22) and arrive at the formulation for the trade-level add-on for transactions in a margined netting set. ${ }^{16}$

$$
\operatorname{AddOn_{i}^{\text {margin}}}(t)=\sigma_{i}(t) \cdot \phi(0) \cdot \sqrt{M P O R}
$$

\subsubsection{Structure of add-on calculations}

The regulatory SA-CCR has a specific structure for the calculation of PFE add-ons. Aggregation procedures are used to calculate netting set level add-ons from tradelevel add-ons. These aggregation rules are based on the central idea that add-ons can be aggregated like standard deviations. While deriving our modified approach for addon calculation, we apply similar assumptions as used for developing the regulatory SA-CCR. We have shown that the general principles of the SA-CCR are still valid under the new target measure $(E E(t))$. Hence, we are generally able to apply the same basic structure and methodology for aggregation as provided by the regulatory SA-CCR.

The first step for calculating the aggregated add-on on netting set level is the determination of an add-on at trade-level. In line with the regulatory SA-CCR, the calculation of trade-level add-ons is asset class specific, but has common features for all derivative transactions. Hence, each transaction is allocated to at least one of five asset classes based on the primary risk factor. ${ }^{17}$ For products with more than one material risk factor, the assignment to multiple asset classes is required. ${ }^{18}$

Following the supervisory SA-CCR, we operate with simple trade-level parameters instead of trade-level volatilities $\left(\sigma_{i}(t)\right)$ directly. Hence, we define a transaction's addon at time $t$ as the product of an exposure factor $\left(E F_{i}\right)$, the adjusted notional amount $\left(d_{i}\right)$, its delta $\left(\delta_{i}\right)$ and a scaling factor with respect to time $(\sqrt{t}$ or $\sqrt{M P O R}) .{ }^{19}$

$$
\begin{array}{r}
\operatorname{AddO}_{i}(t)=E F_{i} \cdot d_{i}(t) \cdot \delta_{i}(t) \cdot \sqrt{t} \\
\operatorname{AddOn}_{i}^{\text {margin }}(t)=E F_{i} \cdot d_{i}(t) \cdot \delta_{i}(t) \cdot \sqrt{M P O R}
\end{array}
$$

By inserting Eq. (21) into Eq. (24) and solving for $\sigma_{i}(t)$, we arrive at the following approximation for the volatility of the transaction's market value at $t$ :

$$
\sigma_{i}(t)=\frac{E F_{i}}{\phi(0)} \cdot d_{i}(t) \cdot\left|\delta_{i}(t)\right|
$$

\footnotetext{
16 As shown in "Appendix A.2", the aggregation of trade-level add-ons also holds true when aggregating margined trade-level add-ons.

17 Within this paper we share the number and set-up of asset classes and hedging sets proposed by the Basel Committee. Nevertheless, the general structure of our approach allows for further modification with respect to the amount and definition of asset classes, hedging and subsets.

18 Details with respect to this requirement are still under discussion. A first discussion paper has been published by EBA (2017).

19 The maturity factor $\left(M F_{i}\right)$ used in the supervisory SA-CCR is applied as correction for trades maturing within the risk horizon of 1 year for unmargined netting sets. Under the target measure $E E(t)$, this is not necessary, as no averaging over a dedicated risk horizon is applied. Hence, a maturity factor is not required.
} 
In accordance with BCBS (2014b) the ratio of $E F_{i}$ and $\phi(0)$ can be interpreted as the annualized standard deviation of the transaction's primary risk factor $\left(\sigma_{i}^{(R F)}\right)$ :

$$
\sigma_{i}^{(R F)}=\frac{E F_{i}}{\phi(0)}
$$

Please note that the volatility of the risk factor $\left(\sigma_{i}^{(R F)}\right)$ is assumed to be constant over time. Hence, the time dependence of the volatility of the transaction's market value is solely resulting from $d_{i}(t)$ and $\delta_{i}(t)$. The exposure factor $\left(E F_{i}\right)$ can be interpreted as an approximation of the expected exposure of a netting set with one directional trade, which has the size of one unit adjusted notional at $t=1$ year.

$$
E F_{i}=\sigma_{i}^{(R F)} \cdot \phi(0)
$$

This relationship allows a calibration of $E F_{i}$ based on the (implied) volatility of the transaction's primary risk factor $\left(\sigma_{i}^{(R F)}\right)$. The supervisory SA-CCR provides supervisory factors $\left(S F_{i}\right)$ on subclass level. ${ }^{20} \mathrm{We}$ introduce a more granular approach to the calibration of the exposure factor in Sect. 4.

The delta parameter $\left(\delta_{i}\right)$ is a function of the direction of the trade with respect to the primary risk factor (long / short). For products with a non-linear relationship to the primary risk factor, $\delta_{i}$ serves as a scaling factor with respect to the moneyness of the product. ${ }^{21}$ For plain vanilla options we use a delta formula based on the formula provided by the supervisory SA-CCR (BCBS 2014c):

$$
\delta_{i}(t)=\psi \cdot N\left(\omega \cdot \frac{\ln (\hat{P}(t) / K)+0.5 \cdot\left(\sigma_{i}^{(i m p l)}\right)^{2} \cdot(T-t)}{\left(\sigma_{i}^{(i m p l)}\right) \cdot \sqrt{(T-t)}}\right)
$$

where $K$ represents the strike price and $\sigma_{i}^{(i m p l)}$ the (implied) volatility of the underlying of the option. $T$ is defined as the amount of time (in years) between today and the expiry date of the option. ${ }^{22} \hat{P}(t)$ equals an estimation of the spot price of the underlying at time $t .^{23}$ If an estimation of $P(t)$ via the forward price is not possible, we assume $P(t)=P\left(t_{0}\right)$. The parameters $\psi$ and $\omega$ are required to cover all combinations of bought/sold and call/put options. ${ }^{24}$

For more complex and exotic options Eq. (29) might not be appropriate as a lot of these products are path-dependent. As $\delta_{i}(t)$ is defined on trade-level, our approach

\footnotetext{
20 Such as rating categories within asset class credit.

21 Please note that supervisory approach is offering a specific delta formula for CDOs which uses detachment and attachment points as inputs for the calculation of the delta parameter.

22 For options with multiple exercise dates, one might only assume the latest exercise date.

23 Example: For FX options, we are able to estimate the forward price at time $t$ based on the interest rate curves of the involved currencies.

$24 \psi$ equals $(-1)$ where the transaction is a sold call option or a bought put option and sign $(+1)$ where the transaction is a bought call option or sold put option. $\omega$ equals $(-1)$ for put and $(+1)$ for call options.
} 
offers the flexibility to include the actual economic deltas for these products. Nevertheless, this requires an assumption on the development of the products delta over time, especially if those deltas are not calculated analytically. Hence, the methodology for calculation of $\delta_{i}(t)$ needs to be defined for each product type based on the availability and quality of the respective data.

The adjusted notional amount $\left(d_{i}\right)$ captures the size of a transaction. For interest rate and credit derivatives, $d_{i}$ is also used to recognize the duration of the instrument and thereby its sensitivity to changes in underlying risk factors. In general, the add-on under SA-CCR is proportional to the adjusted notional. The adjustments to the asset class specific formulation of $d_{i}$ are presented in "Appendix A.3.3".

\subsubsection{Aggregation of trade-level add-ons}

With respect to the aggregation of trade-level add-ons and structure of subsets and hedging sets, we basically follow the procedures and definitions of the supervisory SA-CCR (BCBS 2014b):

In case of interest rate derivatives, all transactions $(i)$ are allocated to a hedging subset based on their currency $(c)$ and maturity. For each currency, three maturity buckets $(0-1 y, 1-5 y,>5 y)$ are defined. Within these maturity buckets $(b)$, tradelevel add-ons of long and short positions are aggregated assuming a correlation of $100 \%$. Hence, we arrive at the following definition for the add-on $(X)$ for each hedging subset $\left(X_{c b}\right)$ at time $t$ :

$$
X_{c b}(t)=\sum_{i \in\left\{c c y_{c}, M B_{b}\right\}} X_{i}(t)=\sum_{i \in\left\{c c y_{c}, M B_{b}\right\}} E F_{i} \cdot d_{i}(t) \cdot \delta_{i}(t) \cdot \sqrt{t}
$$

In a next step we calculate an add-on for each hedging set (currency) based on the following equation:

$$
X_{c}(t)=\sqrt{\sum_{b}\left(X_{c b}(t)\right)^{2}+\sum_{b} \sum_{b \neq d} \rho_{b d} \cdot X_{c b}(t) \cdot X_{c d}(t)}
$$

where $\rho_{b d}$ is defined as the correlation between two maturity buckets. ${ }^{25}$ Given Eq. (31), we are able to account for offsetting effects between long and short transactions in the same currency and different maturity bucket. Based on the adjustments of trade-level parameters $\left(d_{i}(t), \delta_{i}(t)\right)$ over time, we model implicitly the time-variant development of the sensitivity of the transactions and the netting set to changes in the underlying risk factors. Hence, we are able to capture the offsetting effects of long and short positions in the same currency and with different maturities over time.

\footnotetext{
25 In line with BCBS (2014b) the correlation between maturity buckets ( $0-1 \mathrm{y})$ and ( $>5 \mathrm{y})$ is set to $30 \%$, while all correlations between other maturity buckets are set to $70 \%$. Please note, that we assume constant correlation over time in line with our methodological framework (see Eq. (A.4)).
} 
Within the supervisory SA-CCR framework, the regulatory PFE add-on on for IR derivatives on asset class level is defined as the sum of all hedging set add-ons:

$$
X_{r e g}^{(I R)}(t)=\sum_{c} X_{c}(t)
$$

Given the formulation above, the regulatory PFE add-on for IR derivatives does not recognize diversification effects between exposure in different currencies.

The add-on calculation for FX derivatives does not require an allocation of transactions to hedging subsets. All transactions referencing the same currency pair are allocated to a hedging set directly. Within the resulting hedging sets, full offsetting of long and short positions is allowed. Hence, the aggregation of trade-level add-ons $\left(X_{i}\right)$ to the specific hedging set add-on $\left(X_{c}\right)$ for each currency pair $(c)$ can be written as:

$$
X_{c}(t)=\sum_{i \in c c y_{c}} X_{i}(t)=\sum_{i \in c c y_{c}} E F_{i} \cdot d_{i}(t) \cdot \delta_{i}(t) \cdot \sqrt{t}
$$

For FX derivatives, the regulatory PFE add-on on asset class level $\left(X^{(F X)}\right)$ is defined as the simple sum of all hedging set add-ons $\left(X_{c}\right)$ :

$$
X_{r e g}^{(F X)}(t)=\sum_{c} X_{c}(t)
$$

Hence, the regulatory PFE add-on for FX derivatives does not take diversification effects between different currency pairs into account. The aggregated PFE add-on across asset classes is defined as the simple sum of all add-ons on asset class level. Hence, diversification effects across asset classes are also not considered during the aggregation of the PFE add-on.

The aggregation procedures for equity, credit and commodity derivatives involve offsetting of transactions via the application of a single-factor model. Hence, offsetting of transactions with different underlying reference entity $(\mathrm{CR})$, issuer (EQ) or commodity type (COM) is considered, when calculating the PFE add-on. ${ }^{26}$

In general, the recognition of diversification effects in the add-on calculation of the supervisory SA-CCR is deemed conservative. We perceive the missing consideration of offsetting effects across currencies (IR) and currency pairs (FX) as too conservative for the purpose of CVA calculation. Our approach provides the flexibility for implementing improvements to the methodology for the aggregation of PFE add-ons. As a simple example we introduce a modified aggregation procedure for the calculation of IR and FX add-ons on asset class level. This method is based on the new standardized approach for market risk (BCBS 2019), where this concept is used to aggregate bucket level results ( $\mathrm{FX}=$ currency pairs, $\mathrm{IR}=$ currencies) to asset class results. Following BCBS (2019), we define the asset class level add-on of the modified SA-CCR for asset classes FX and IR as:

\footnotetext{
${ }^{26}$ For further details on the aggregation procedures for those asset classes, we refer to BCBS (2014b) and BCBS (2014c).
} 


$$
X_{\bmod }^{(I R / F X)}(t)=\sqrt{\sum_{b}\left(X_{b}(t)\right)^{2}+\sum_{b} \sum_{b \neq c} \gamma_{b c}^{(a)} \cdot X_{b}(t) \cdot X_{c}(t)}
$$

where $X_{b}(t)$ equals the add-on of the respective hedging set $(b) . \gamma_{b c}$ represents the correlation between two currency pairs (FX) or currencies (IR). In line with BCBS (2019) we set $\gamma_{b c}^{(I R)}=0.5$ and $\gamma_{b c}^{(F X)}=0.6$. In the modified SA-CCR, Eq. (35) is used to replace Eqs. (32) and (34).

As mentioned above, the modified SA-CCR framework offers the possibility to include more complex aggregation methodologies to model correlations between hedging sets and asset classes. The derivation of more complex modified procedures for the aggregation of add-ons would require a comprehensive discussions and analysis of different aggregation methods for each asset class. Within this paper we focus on providing detailed insights in the methodological foundations of the modified SA-CCR. Hence, we choose a simple aggregation methodology to account for diversification effects between hedging sets in the asset classes IR and FX.

\subsection{Multiplier}

When deriving the PFE multiplier, the assumptions AO1 and AO2 are relaxed. Hence, the market value of a netting set can be different to zero and received or posted collateral might be present. The multiplier is defined as a fraction of PFE. Thereby the PFE is corrected for the fact that market value and collateral amounts are different from zero. Based on these assumptions, the expected exposure $(E E(t))$ of an unmargined netting set $(k)$ at a certain point in time $t$ is defined as:

$$
E E_{k}(t)=\mathbb{E}^{Q}\left[\max \left(\left(\hat{V}_{k}(t)+\sigma_{k}(t) \cdot \sqrt{t} \cdot Y\right)-C_{C E}(t), 0\right)\right]
$$

where:

- $\hat{V}_{k}(t)$ is the (deterministic) market value of the netting set at time $t$,

$-C_{C E}(t)$ represents the cash-equivalent value of net collateral received at $t$,

$-\sigma_{k}(t)$ is the volatility of the netting set at time $t$ and

- $Y$ is defined as a standard normal random variable.

Based on Eq. (36) and the aforementioned assumptions, the multiplier formula is derived analytically for unmargined netting sets. For details on assumptions and analytical calculation of the multiplier formula, please refer to "Appendix A.3". The multiplier for a netting set $(k)$ at time $t$ is defined as:

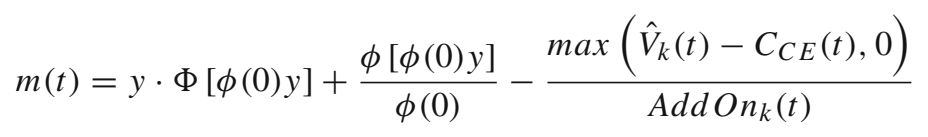

where $y=\frac{\hat{V}_{k}(t)-C_{C E}(t)}{\operatorname{Add} O n_{k}(t)}, \Phi($.$) is the standard normal cumulative distribution function$ and $\phi($.$) the standard normal probability density function. This differs from the model-$ based multiplier formula derived by BCBS (2014b). The supervisory SA-CCR applies 
a more conservative multiplier function to account for the possibility that future MtM values are not normally distributed. Additionally only cases are considered where $\hat{V}_{k}(t)-C_{C E}(t)$ is less than zero. Futhermore, a floor is introduced in order to prevent the multiplier from reaching zero. The modified SA-CCR uses the multiplier as defined in Eq. (37) without further modifications. As shown in "Appendix A.3", the same formulation applies for margined and unmargined netting sets.

\section{Calibration}

\subsection{Background and general considerations}

In its regulatory setup, the SA-CCR is calibrated under the real-world measure based on historical data. According to BCBS (2013) the regulatory SA-CCR parameters were estimated using a three step approach. In a first step, the supervisory parameters were calibrated based on market data from different markets. Volatilities and correlations were evaluated based on a stress period which, in most cases, was defined as the three-year period with the largest historically observed volatility. The BCBS applied different approaches by asset class to perform this initial calibration. ${ }^{27}$ The second step was based on a comparison of SA-CCR exposure outcomes with results from simplified IMM models for a set of hypothetical portfolios. This comparison was carried out for small portfolios involving hypothetical trades for each asset class. The third and final step involved a benchmarking exercise based on contributions by a set of IMM banks via Quantitative Impact Studies. The model outcomes were averaged and compared with CEM and SA-CCR exposures to derive final adjustments to the regulatory parameters.

The outcome of this process is regulatory prescribed parameters (option volatilities, supervisory factors and correlations) for each asset class. ${ }^{28}$ For some asset classes, such as interest rates or foreign exchange, the supervisory factor is defined on asset class level. Hence, there is no differentiation between more granular risk factors such as currencies or tenors. For some asset classes additional levels (subclasses) were introduced to increase the granularity and risk sensitivity of the approach. ${ }^{29}$ The BCBS tried to limit the granularity of the regulatory approach, aiming for a total number of risk factors close to the Current Exposure Method (CEM). As the SA-CCR was not designed to cover exotic products or more complex risk factors, a certain degree of conservatism was included when developing the approach and defining model parameters (BCBS 2013).

The supervisory parameters given by the BCBS are not appropriate when generating exposure profiles for accounting and pricing purposes. In summary, the main issues are the calibration under the real-world measure based on historical (stressed) volatilities, the lack of granularity with respect to risk factors, as well as the high degree of

\footnotetext{
27 For a detailed overview on the calibration by asset class, please refer to BCBS (2013).

28 The supervisory parameters are available in BCBS (2014c).

29 Example: For credit derivatives, the supervisory factor is defined based on the underlying type (index or single-name) and the underlying's credit quality.
} 
conservatism applied to the overall calibration approach. Hence, a re-calibration of the parameters is required in order to use the modified SA-CCR for CVA calculation.

\subsection{Calibration of the modified SA-CCR}

The calibration of the modified SA-CCR has two main objectives. First, we aim to increase the granularity of risk factors aiming for more risk sensitive exposure calculation. Second, the calibration is performed under the risk-neutral measure based on market-implied volatilities, in order to meet the expectation of market participants. Overall, the main driver of exposure is the volatility of the primary risk factor of each transaction. Hence, we focus on the calibration of the exposure factor $\left(E F_{i}\right)$. We do not provide a re-calibration of the SA-CCR correlation parameters, but apply a modified methodology for the aggregation of hedging set add-ons involving additional correlation parameters (see Sect. 3.2.4). We perform a calibration of option volatilities for the calculation of the delta parameter (see Eq. (29)). The calibration approach differs by asset class and depends on data availability for the respective risk factors. Within this section, we provide a general overview and present proposals for the calibration of the modified SA-CCR, without discussing all asset class specific details. We focus on the asset classes IR and FX, as these are relevant for the subsequent empirical analysis.

In general, the calibration of exposure factors is based on market-implied volatilities obtained from options. Hence, the approach offers the possibility to consider the maturity and the moneyness of a certain position. For interest rate derivatives, the exposure factor is calibrated based on market-implied at-the-money (ATM) swaption volatilities for the respective currency, taking the volatility term structure into account. Hence, the risk factor is defined by the combination of currency and tenor. In case of missing data we propose to use the supervisory factor $(0.5 \%)$.

In line with BCBS (2013) we calibrate the exposure factor for FX derivatives directly from implied FX option volatilities using the relationship stated in Eq. (28). While the supervisory SA-CCR only applies one distinct supervisory factor for the whole FX asset class, we consider each currency pair a single risk factor. Hence, the volatility used to calculate the exposure factor for a specific transaction is a function of its base and reference currency. The market-implied ATM volatilities are provided based on the following term structure: $1 \mathrm{D}, 1 \mathrm{~W}, 1 \mathrm{M}, 2 \mathrm{M}, 3 \mathrm{M}, 6 \mathrm{M}, 9 \mathrm{M}, 12 \mathrm{M}, 2 \mathrm{Y}, 3 \mathrm{Y}, 4 \mathrm{Y}, 5 \mathrm{Y}$, $10 \mathrm{Y}$. In order to calculate the relevant volatility value for a specific transaction, we use linear interpolation. If there is no valid and appropriate data on implied volatilities for a certain currency pair, we use the supervisory factor $(4 \%)$.

For other asset classes (Equity, Credit, Commodity) the granularity of exposure factors can be chosen based on available data and desired complexity of implementation. For equity and credit derivatives, the calibration for each underlying can be carried out independently or via a beta-approach as described in BCBS (2013), where exposure factors for single-name positions are obtained from index volatilities and the respective beta. For commodity positions, different dimensions, such as underlying, grade and delivery location can be considered when calibrating exposure factors. In general, one could also decide to calibrate the exposure factor on broad commodity types similar to the supervisory approach. By maintaining the key building blocks of 
the regulatory SA-CCR, we provide a flexible framework for the calibration of the modified SA-CCR. This leads to a general trade-off between the risk-sensitivity of the approach and the amount of data required for its calibration.

\section{Empirical analysis}

\subsection{Methodology}

In this section we assess the modified SA-CCR's ability to provide a risk sensitive and accurate approximation of the exposure calculated by an advanced model. As the main criterion, we compare the resulting credit valuation adjustment (CVA) from both approaches. The analysis is performed based on illustrative examples of netting sets. These are composed of hypothetical interest rate (IR) and foreign exchange (FX) transactions. The netting sets involve products with different maturity, underlying, direction and moneyness. Our empirical study comprises the following main steps:

$S A-C C R$ (re)calibration: First, we calibrate the relevant exposure factors $\left(E F_{i}\right)$ of the modified SA-CCR to market-implied volatilities as described in Sect. $4 .{ }^{30} \mathrm{We}$ construct a series of netting sets for the empirical analysis including margined and unmargined netting sets as well as netting sets with non-linear products. An overview of the transactions and netting sets is provided in "Appendix A.4.2". The calibration of the relevant parameters is carried out based on a market data set as of 28th September 2018.

Exposure calculation: Second, we calculate $E E_{k}(t)$ based on the modified SACCR and an advanced benchmark model (BMM). For interest rates, we apply a model based on the approach of Trolle and Schwartz (2009). The modelling of FX rates is based on a Heston model (1993). The parameters of the Trolle-Schwartz model are calibrated to European swaptions. The calibration process is based on swaption prices derived from quoted implied volatilities for a series of European swaptions with different underlying and option tenor as well as different strike price. Parameters of the Heston model are calibrated based on implied lognormal volatilities quoted for FX option strategies. Quotes are available for different maturity and moneyness. These strategies are transformed to European FX options and their corresponding prices, which are used as input for the calibration process. ${ }^{31}$

From our point of view, the applied models provide state-of-the-art stochastic processes for the evolution of all major risk factors. The models also cover the dependency of the implied volatility to moneyness (volatility skew). Accuracy is a critical issue when calculating exposures for CVA pricing and accounting purposes. Hence, we decided to use an advanced, state-of-the-art benchmark model involving a comprehensive set of risk factors to assess the accuracy of the modified SA-CCR, rather than a more simplistic exposure model.

CVA calculation: Based on the exposure profiles obtained from the modified SACCR and the benchmark model, we calculate the CVA using the following discretized

\footnotetext{
${ }^{30}$ Please note that other parameters, such as correlations, are not re-calibrated. Hence, we use the supervisory parameters given by the regulatory SA-CCR.

${ }^{31}$ For additional details on the calibration of the applied benchmark model please refer to "Appendix A.4.1".
} 
formula under the assumption of a flat credit spread curve (40 basis points) and a recovery rate of $40 \%$ (Gregory 2010 ).

$$
C V A \approx(1-R) \cdot \sum_{i=1}^{M} D F\left(t_{i}\right) \cdot E E\left(t_{i}\right) \cdot P D\left(t_{i-1}, t_{i}\right)
$$

Analysis: Finally, we compare the exposure profiles and the CVA results for the benchmark model with the modified SA-CCR. The targets of this comparison are the assessment of the applicability for the purpose of CVA calculation, the validation of results as well as the identification of shortcomings and areas of future work. Hence, we use illustrative examples for different products and situations.

\subsection{Results}

\subsubsection{Results for interest rate swaps}

The expected exposure profile of an IR swap is driven by two contrary effects. The uncertainty with respect to future payments leads to an increasing exposure (dispersion), whereas the roll-off of swap payments has a decreasing effect on the exposure (amortization) over time. The combination of these effects results in the typical humped shape of the exposure profile (Gregory 2010). Within the modified SA-CCR, the dispersion effect is reflected by scaling the volatility with the square root of time $(\sqrt{t})$ when calculating the PFE add-on. ${ }^{32}$ As shown above, the modified SA-CCR approach is also able to recognize the amortization effect due to the consideration of cash flows in replacement costs and the adjustment of the duration parameter, which is used to calculate the PFE add-on. Our approach allows the generation of expected (positive) exposure $(E E)$, expected negative exposure $(E N E)$ and expected market value profiles $(E M t M) .{ }^{33}$ These different types of exposure profiles are presented in Fig. 1 for an EUR 5Y at-the money (ATM) IR payer swap, with differing payment frequencies (fix = annual, float $=$ semi-annual).

The shape of the exposure profiles modelled with the modified SA-CCR is in line with the results of the advanced benchmark model (BMM). By calibrating the modified SA-CCR approach to market-implied volatilities, we are able to reflect a similar level of risk. Additionally, Fig. 1 shows that the modified SA-CCR is able to recognize the asymmetry between payer and receiver swaps, as well as the differing payment frequencies of the fixed and floating leg. By using an adjusted SA-CCR multiplier formula, we consider the effect of the current market value on future exposure uncertainty. Figure 2 provides expected exposure profiles and corresponding CVA results of EUR 10Y ATM IR payer swaps with different moneyness.

The results show that our approach mirrors the exposure dynamics produced by an advanced model. Based on the generated exposure profiles, we are able to calcu-

\footnotetext{
32 See Eq. (21).

33 Please note that the following presentation of results is focused on the calculation of CVA and thereby the generation of expected (positive) exposure $(E E)$.
} 

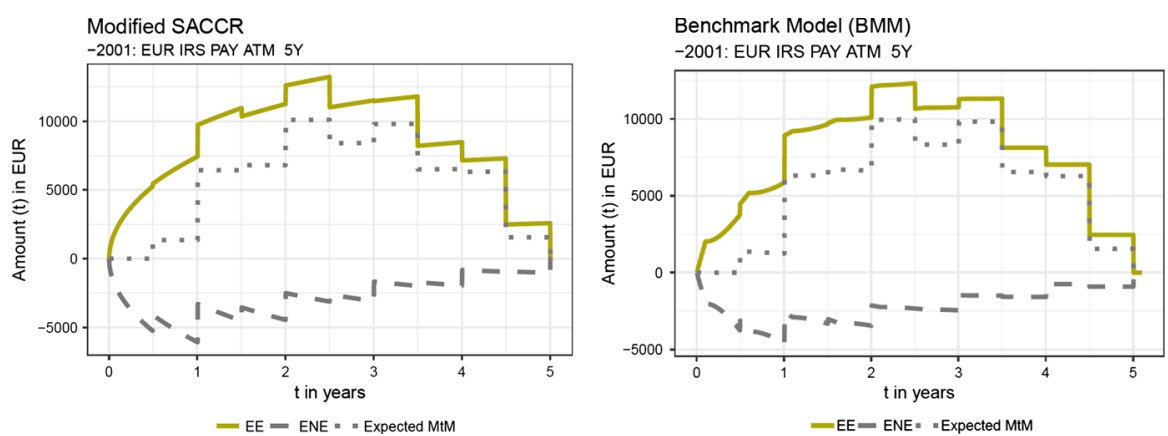

Fig. 1 Exposure profiles of an EUR 5Y ATM IR (payer) swap. Note The figure shows different types of exposure profiles for an EUR 5Y ATM IR (payer) swap, calculated with the modified SA-CCR approach (left panel) and a benchmark model (right panel)
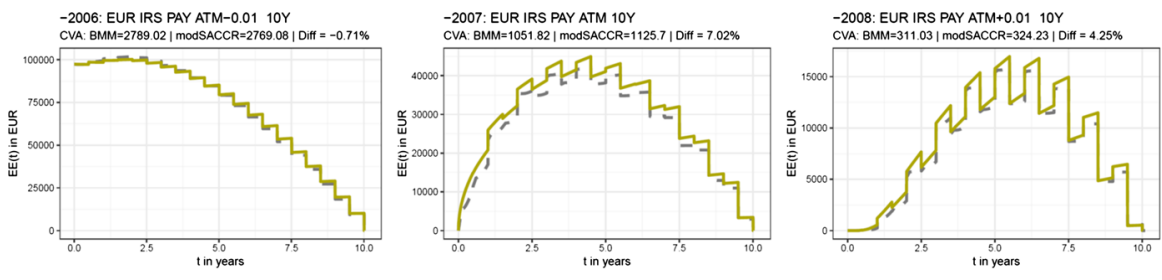

Fig. 2 Expected exposure of EUR 10Y IR payer swaps. Note The figure shows the expected exposure profile $(E E(t))$ of IR (payer) swaps with a maturity of 10 years, which are in-the-money (left), at-themoney (middle) and out-of-the-money (right). The results of the modified SA-CCR (solid line) are close to the results retrieved from the benchmark model (dashed line). This is also indicated by the corresponding CVA results shown in the header of each graph

late Credit Valuation Adjustments (CVA) by using Eq. (38) and the aforementioned assumptions with respect to the credit spread curve and recovery rate (see Sect. 5.1).

In order to assess the quality of the approximation by the modified SA-CCR, we apply the approach to an illustrative set of IR swaps with different underlying currencies, tenors and moneyness. We use 14 hypothetical IR swap transactions. Each transaction is put into a separate uncollateralised and unmargined netting set. All hypothetical transactions are fix-to-floating IR swaps with identical payment frequencies (fixed $=$ anual, float $=$ semi-annual). The test data comprises swaps with different underlying currencies (EUR, USD), tenors (5Y, 7Y, 10Y), direction (pay, receive) and moneyness (ATM+0.01,ATM, ATM-0.01). ${ }^{34}$ Table 1 provides an overview of the CVA results for the different netting sets. ${ }^{35}$

In general, we observe that the expected market value estimated by the modified SACCR is very close to the results from the benchmark model for all analysed netting sets. This is a result of the cash flow based calculation of replacement costs. The excellent approximation of the expected market value leads to very good results for in-themoney (ITM) transactions, as their future exposure is mainly driven by movements

\footnotetext{
34 For additional details on the hypothetical transactions, please please refer to "Appendix A.4.2".

35 A graphical representation of the expected exposure profiles for these netting sets are provided in "Appendix A.4.3".
} 
Table 1 CVA results for hypothetical IR swaps

\begin{tabular}{llll}
\hline Id & Description & CVA (SA-CCR) & CVA (BMM) \\
\hline-2000 & EUR IRS PAY ATM-0.01 5Y & 703.82 & 702.95 \\
-2001 & EUR IRS PAY ATM 5Y & 167.68 & 155 \\
-2002 & EUR IRS PAY ATM+0.01 5Y & 11.55 & 11.21 \\
-2003 & EUR IRS REC ATM-0.01 5Y & 0.52 & 0.12 \\
-2004 & EUR IRS REC ATM 5Y & 55.22 & 43.57 \\
-2005 & EUR IRS REC ATM+0.01 5Y & 490.03 & 491.33 \\
-2006 & EUR IRS PAY ATM-0.01 10Y & 2769.08 & $2,789.02$ \\
-2007 & EUR IRS PAY ATM 10Y & 1125.70 & $1,051.82$ \\
-2008 & EUR IRS PAY ATM+0.01 10Y & 324.23 & 311.03 \\
-2009 & EUR IRS PAY ATM-0.01 7Y & 1370.16 & $1,363.46$ \\
-2010 & EUR IRS PAY ATM 7Y & 410.23 & 400.67 \\
-2011 & EUR IRS PAY ATM+0.01 7Y & 57.68 & 69.2 \\
-2012 & USD IRS PAY ATM-0.01 5Y & 470.25 & 457.78 \\
-2013 & USD IRS PAY ATM 5Y & 120.95 & 96.75 \\
-2014 & USD IRS PAY ATM+0.01 5Y & 20.20 & 13.2 \\
\hline
\end{tabular}

This table provides an overview of the CVA results for different hypothetical IR swaps calculated with (1) the modified SA-CCR approach and (2) the benchmark model (BMM). We provide a graphical representation of the expected exposure profile for each netting set in "Appendix A.4.3"

in the expected MtM (see table 1). Given the granular calibration approach of the modified SA-CCR, we are able to consider different levels of risk with respect to the underlying currency and tenor of the transactions. The calibration to at-the-money (ATM) market-implied volatilities leads to an appropriate approximation for most netting sets, even if the respective transaction is not at-the-money (see Fig. 2 and Table 1).

The modified SA-CCR is able to provide an adequate and risk sensitive approximation of the expected exposure profile for IR swaps. We are able to use these as input for the calculation of CVA and receive very good results on single-transaction level. The approach is capable of reflecting all major exposure dynamics and estimating a reasonable level of risk. Furthermore, the modified SA-CCR is sensitive to different underlying currencies, tenors and the moneyness of transactions.

\subsubsection{Results for FX forwards}

The risk of FX forwards is dominated by the notional exchange at maturity $(T)$ of the transaction. FX forwards do not involve any further cash flows between $(t)$ and $(T)$. Hence, the exposure is driven by the uncertainty regarding future payments at maturity. The exposure monotonically increases with time and is also driven by small effects from interest rate risk (Gregory 2010). We are able to recognize these characteristics in the modified SA-CCR via a cash flow based calculation of replacement costs as well as the scaling of the add-on by $\sqrt{t}$ (see equation 24). Figure 3 provides the expected exposure profile of $3 \mathrm{Y}$ EUR/USD FX forwards with different moneyness. 

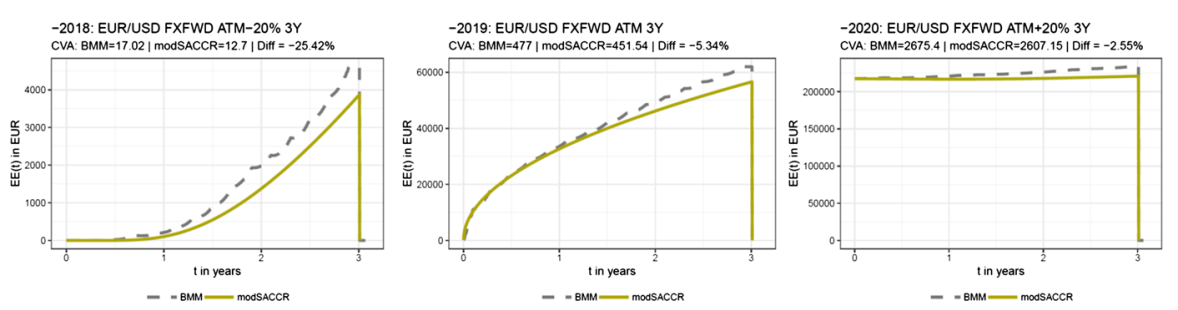

Fig. 3 Expected exposure profile for FX forwards with different moneyness. Note The figure shows the expected exposure profile and CVA results for three EUR/USD FX forwards with maturity of 3 years and different moneyness. The results of the modified SA-CCR (solid line) are very close to the results from the benchmark model (dashed line) for ITM and ATM transactions

The results presented in Fig. 3 show that the modified SA-CCR reflects the influence of current market values on the uncertainty of future exposures. Furthermore, the shape of the generated exposure profiles are in line with the outcome of the benchmark model. Based on the resulting expected exposure profiles, we are able to calculate the CVA for different products and netting sets. For an illustrative validation of the modified SACCR, we have composed 18 single-transaction nettings sets. Furthermore, we assume the absence of collateral and margin agreements. The netting sets are comprised of FX forwards with different maturity, underlying currency pair, as well as moneyness. Table 2 provides an overview of the CVA results for these hypothetical netting sets. ${ }^{36}$

In general, the expected exposure of FX forwards is more sensitive to calibration compared to IR swaps. As discussed, the risk of FX forwards is concentrated on payments at maturity. Hence, the uncertainty of future exposure increases over time. There are no exposure-reducing effects on the expected exposure from roll-off of payments during the life-time of the transaction. Hence, ATM and OTM transactions are very sensitive to uncertainty in calibration. In contrast, ITM transactions are less sensitive to calibration, as the expected exposure is mainly driven by the current and expected market value. Hence, the modified SA-CCR generates reasonable expected exposure profiles for ITM transactions, which are very close to the outcome of the benchmark model. The expected exposure of OTM transactions is mainly driven by the PFE add-on. Hence, results are more sensitive to calibration of the add-on. As we calibrate the modified SA-CCR to ATM volatilities, we are not able to correctly reflect the level of risk estimated by the benchmark model for all currencies and tenors.

Nevertheless, the modified SA-CCR considers the exposure mitigating effect of current negative market values and is able to reproduce the shape of the exposure profile. Hence, an advanced calibration approach considering the dependency of marketimplied volatility on moneyness should lead to more appropriate results. For ATM FX forwards, the modified SA-CCR provides reasonable exposure profiles and an appropriate approximation of the CVA estimated by the benchmark model.

Taking the results for the illustrative examples into account, we are justified in saying that our approach is able to provide an expected exposure profile, which could serve as a reasonable basis for the approximation of the CVA. The approach incorporates the specific risks of different currency pairs and tenors. Furthermore, the risk

$\overline{36}$ The visualization of $E E(t)$ profiles is available in "Appendix A.4.4". 
Table 2 CVA results for hypothetical FX forwards

\begin{tabular}{llll}
\hline Id & Description & CVA (SA-CCR) & CVA (BMM) \\
\hline-2015 & EUR/USD FXFWD ATM-20\% 1Y & 0.05 & 0.08 \\
-2016 & EUR/USD FXFWD ATM 1Y & 81.41 & 80.19 \\
-2017 & EUR/USD FXFWD ATM+20\% 1Y & 827.75 & 827.96 \\
-2018 & EUR/USD FXFWD ATM-20\% 3Y & 12.70 & 17.02 \\
-2019 & EUR/USD FXFWD ATM 3Y & 451.54 & 477.00 \\
-2020 & EUR/USD FXFWD ATM+20\% 3Y & $2,607.15$ & 2675.40 \\
-2021 & EUR/JPY FXFWD ATM-20\% 3Y & 41.98 & 27.41 \\
-2022 & EUR/JPY FXFWD ATM 3Y & 548.06 & 509.16 \\
-2023 & EUR/JPY FXFWD ATM+20\% 3Y & $2,431.10$ & 2538.73 \\
-2024 & EUR/GBP FXFWD ATM-20\% 3Y & 22.71 & 45.89 \\
-2025 & EUR/GBP FXFWD ATM 3Y & 487.13 & 462.00 \\
-2026 & EUR/GBP FXFWD ATM+20\% 3Y & 2489.46 & 2495.68 \\
-2027 & EUR/CHF FXFWD ATM-20\% 1Y & 0.01 & 0.45 \\
-2028 & EUR/CHF FXFWD ATM 1Y & 67.95 & 64.50 \\
-2029 & EUR/CHF FXFWD ATM+20\% 1Y & 800.52 & 799.50 \\
-2030 & USD/GBP FXFWD ATM-20\% 3Y & 41.07 & 83.08 \\
-2031 & USD/GBP FXFWD ATM 3Y & 559.32 & 595.09 \\
-2032 & USD/GBP FXFWD ATM+20\% 3Y & 2486.28 & 2369.26 \\
\hline This & ES & & \\
\hline
\end{tabular}

This table provides an overview of the CVA results for different hypothetical FX forwards calculated with (1) the modified SA-CCR approach and (2) the benchmark model (BMM). We provide a graphical representation of the expected exposure profile for each netting set in "Appendix A.4.4"

mitigating effect of current negative market values is considered via the PFE multiplier. Nevertheless, we have uncovered calibration issues with respect to OTM transactions. These could be solved by taking the dependency of the market-implied volatility to the moneyness of the transaction into account.

\subsubsection{Results for multi-transaction netting sets}

In addition to the analysis of single transactions, we apply our approach to illustrative multi-transaction netting sets. The supervisory SA-CCR provides a holistic framework for the aggregation of trade-level add-ons across hedging (sub)sets and asset classes. The supervisory methodology is considered to be conservative, as it proscribes institutions to recognize diversification effects between risk factors which are considered to be correlated in some cases (e.g. interest rate risk in different currencies). ${ }^{37}$ As stated in Sect. 3.2.4, we apply an adjusted aggregation procedure for the aggregation of hedging set add-ons to results on asset class level using regulatory correlation parameters introduced in BCBS (2019). Hence, our approach is still expected to produce conservative results for netting sets with transactions in different hedging sets.

\footnotetext{
37 For more detailed information on the aggregation mechanisms, please refer to BCBS (2014b, 2014c).
} 
Table 3 CVA results for hypothetical combined netting sets

\begin{tabular}{|c|c|c|c|c|}
\hline Id & Trade Id & Description & CVA (SA-CCR) & CVA (BMM) \\
\hline \multirow[t]{2}{*}{-2033} & -2001 & EUR IRS PAY ATM 5Y & 0.00 & 0.00 \\
\hline & -2004 & EUR IRS REC ATM 5Y & & \\
\hline \multirow[t]{3}{*}{-2034} & -2001 & EUR IRS PAY ATM 5Y & 1509.91 & 1616.82 \\
\hline & -2007 & EUR IRS PAY ATM 10Y & & \\
\hline & -2010 & EUR IRS PAY ATM 7Y & & \\
\hline \multirow[t]{2}{*}{-2035} & -2013 & USD IRS PAY ATM $5 Y$ & 236.43 & 113.26 \\
\hline & -2001 & EUR IRS PAY ATM $5 Y$ & & \\
\hline \multirow[t]{3}{*}{-2036} & -2012 & USD IRS PAY ATM-0.01 5Y & 362.86 & 290.24 \\
\hline & -2013 & USD IRS PAY ATM 5Y & & \\
\hline & -2014 & USD IRS PAY ATM+0.01 5Y & & \\
\hline \multirow[t]{2}{*}{-2037} & -2019 & EUR/USD FXFWD ATM 3Y & 895.19 & 730.41 \\
\hline & -2022 & EUR/JPY FXFWD ATM 3Y & & \\
\hline \multirow[t]{2}{*}{-2038} & -2019 & EUR/USD FXFWD ATM 3Y & 462.59 & 415.14 \\
\hline & -2031 & USD/GBP FXFWD ATM 3Y & & \\
\hline \multirow[t]{2}{*}{-2039} & -2028 & EUR/CHF FXFWD ATM $1 Y$ & 609.61 & 591.44 \\
\hline & -2031 & USD/GBP FXFWD ATM 3Y & & \\
\hline \multirow[t]{2}{*}{-2040} & -2019 & EUR/USD FXFWD ATM 3Y & 532.95 & 535.98 \\
\hline & -2016 & EUR/USD FXFWD ATM 1Y & & \\
\hline \multirow[t]{2}{*}{-2041} & -2019 & EUR/USD FXFWD ATM 3Y & 501.31 & 491.82 \\
\hline & -2004 & EUR IRS REC ATM 5Y & & \\
\hline
\end{tabular}

This table provides an overview of the CVA results for different hypothetical netting sets with multiple transactions, calculated with (1) the modified SA-CCR approach and (2) the benchmark model (BMM). We provide a graphical representation of the expected exposure profile for each netting set in "Appendix A.4.5"

The hypothetical netting sets are designed to cover the aggregation across different elements of the SA-CCR (e.g hedging sets, asset classes, sub-hedging sets). In total, we examine nine hypothetical netting sets. ${ }^{38}$ Table 3 provides an overview of the CVA results and the composition for these netting sets.

Nettings sets 2033, 2034 and 2036 are composed of IR derivatives referencing the same underlying currency, while netting set 2035 comprises two IR swaps with different currencies. For netting set 2033, the expected exposure profile is considered to be zero, as the involved transactions have the same currency and tenor, but the opposite direction. We are allowed to fully offset the expected exposure of these transactions resulting in an expected exposure and CVA of zero. The results for netting set 2034 show that the aggregation procedures of the supervisory SA-CCR produce an ade-

38 Details on the composition of theses netting sets are provided in "Appendix A.4.2". 
quate result for multiple transactions in the same currency, but with different maturity. The exposure of netting set 2035 is over-estimated. This results from the obviously insufficient recognition of diversification effects between different currencies via the modified aggregation formula (see Eq. (35)).

When calculating the FX add-on, the modified SA-CCR allows netting between different currency pairs. Nevertheless, the expected exposures for netting sets 2037 , 2038 and 2039 are slightly overestimated. The result for netting set 2040 shows, that our approach is suitable for netting sets composed of transactions with the same underlying currency, but different tenors. Netting set 2041 provides an illustrative example for a portfolio with transactions in multiple asset classes. The SA-CCR does not allow the recognition of netting effects across asset classes when calculating the add-on. Nevertheless, the CVA results for netting set 2041 are very close to the outcome of the benchmark model.

The results for multi-transaction netting sets affirm that the aggregation methodology is an important element of the modified SA-CCR. Especially, the aggregation across different currencies without recognition of offsetting effects is a critical issue with respect to the accuracy of the modified SA-CCR. Hence, there needs to be additional work on the incorporation of diversification effects between hedging sets. The aggregation across asset classes does not seem to be a major issue for netting sets composed of IR and FX transactions. Notwithstanding, it should be mentioned that the illustrative examples only cover a limited number of compositions and situations. To fully assess the applicability of the modified SA-CCR, it is necessary to extend the empirical analysis to additional examples based on hypothetical and real-world data.

\subsubsection{Results for collateralized portfolios}

In order to assess the treatment of collateral in the modified SA-CCR, we calculate CVA for 6 selected netting sets based on the assumption of a perfect CSA. ${ }^{39}$ The respective netting sets have already been presented in the previous sections on the results of IR swaps and FX forwards. The assumption of a perfect CSA leads to replacement costs of zero and a PFE multiplier of 1 as the amount of collateral is equal to the market value of the netting set at all future points in time $t$. Hence, the expected exposure under the modified SA-CCR is solely driven by the PFE add-on, which is calculated for a risk horizon equal to the margin period of risk.

Table 4 provides an overview on the CVA results for the selected netting sets under the assumption of a perfect CSA. The results show that the modified SA-CCR is capable of capturing the effects from margining on the expected exposure and the resulting CVA. For FX forwards $(2015,2016,2017)$ we receive nearly identical results compared to the benchmark model. The results of the modified SA-CCR do not differ by moneyness as the market value is fully collateralized and therefore not impacting the replacement costs or the PFE multiplier. With respect to IR swaps, we recognize a difference in the CVA results. Nevertheless, we are able to mirror the key exposure dynamics produced by the BMM (see "Appendix A.4.6").

\footnotetext{
39 The existence of a perfect CSA implies: $T H=0, M T A=0$, absence of rounding, no inital margin and instantaneous collateral exchange.
} 
Table 4 CVA results for collateralized portfolios (perfect CSA)

\begin{tabular}{llll}
\hline Id & Description & CVA (SA-CCR) & CVA (BMM) \\
\hline-2000 & EUR IRS PAY ATM-0.01 5Y & 16.33 & 10.47 \\
-2001 & EUR IRS PAY ATM 5Y & 16.33 & 10.64 \\
-2002 & EUR IRS PAY ATM+0.01 5Y & 16.33 & 10.83 \\
-2015 & EUR/USD FXFWD ATM-20\% 1Y & 24.17 & 24.02 \\
-2016 & EUR/USD FXFWD ATM 1Y & 24.17 & 23.98 \\
-2017 & EUR/USD FXFWD ATM+20\% 1Y & 24.17 & 23.94 \\
\hline
\end{tabular}

This table provides an overview of the CVA results for different hypothetical netting sets under the assumption of a perfect CSA calculated with (1) the modified SA-CCR approach and (2) the benchmark model (BMM). We provide a graphical representation of the expected exposure profile for each netting set in "Appendix A.4.6"

Table 5 CVA results for collateralized portfolios (CSA: TH=5.000, MTA=1.000)

\begin{tabular}{llll}
\hline Id & Description & CVA (SA-CCR) & CVA (BMM) \\
\hline-2000 & EUR IRS PAY ATM-0.01 5Y & 99.2 & 108.52 \\
-2001 & EUR IRS PAY ATM 5Y & 79.71 & 63.98 \\
-2002 & EUR IRS PAY ATM+0.01 5Y & 0.76 & 0.58 \\
-2015 & EUR/USD FXFWD ATM-20\% 1Y & 15.43 & 15.97 \\
-2016 & EUR/USD FXFWD ATM 1Y & 24.51 & 30.85 \\
-2017 & EUR/USD FXFWD ATM+20\% 1Y & 35.55 & 43.96
\end{tabular}

This table provides an overview of the CVA results for different hypothetical netting sets under the assumption of an imperfect CSA $(T H=5.000, M T A=1.000)$ calculated with (1) the modified SA-CCR approach and (2) the benchmark model (BMM). We provide a graphical representation of the expected exposure profile for each netting set in "Appendix A.4.7"

In addition to the analysis of netting sets under the assumption of a perfect CSA, we consider the same netting sets given an imperfect CSA $(T H=5.000, M T A=1.000)$. The following calculations are preformed under the assumption of instantaneous fulfilment of collateral calls. Table 5 provides an overview on the CVA results for the selected netting sets under the assumption of an imperfect CSA.

The results for FX forwards are basically in line with the results from the BMM. Nevertheless, there are differences with respect to the level of expected exposure. These differences are mainly driven by the fact that the expected market value of the modified SA-CCR differs slightly from the BMM. The expected future market value of the modified SA-CCR does not consider all risk factors, such as cross-currency basis spreads. Hence, the market value differs from the BMM. The difference in the expected market value leads to a different collateral path. These effects result in a different consideration of collateral and CVA values. The results for IR swaps are also well in line with the CVA values from the BMM. The modified SA-CCR is able to capture the combination of collateral parameters and the decreasing duration of an IR swap over time. In summary, we are certainly justified in saying that the modified SA-CCR is able to capture the main exposure dynamics of margined netting sets. Nevertheless, 
Table 6 CVA results for FX options

\begin{tabular}{llll}
\hline Id & Description & CVA (SA-CCR) & CVA (BMM) \\
\hline-2126 & EUR/USD FX call option ATM (1Y) & 127.92 & 124.34 \\
-2128 & EUR/USD FX put option ATM (1Y) & 127.26 & 115.86 \\
-2130 & EUR/USD FX call option ITM (1Y) & 801.10 & 807.98 \\
-2131 & EUR/USD FX put option OTM (1Y) & 8.97 & 0.12 \\
-2132 & EUR/USD FX call option OTM (1Y) & 0.12 & 0.91 \\
-2133 & EUR/USD FX put option ITM (1Y) & 801.10 & 803.44 \\
\hline
\end{tabular}

This table provides an overview of the CVA results for different hypothetical FX options calculated with (1) the modified SA-CCR approach and (2) the benchmark model (BMM). We provide a graphical representation of the expected exposure profile for each netting set in "Appendix A.4.8"

there is room for additional analysis, validation and potential improvements of the collateral treatment.

\subsubsection{Results for non-linear products}

In addition to the presented examples, we analyse the treatment of non-linear products. For this purpose, we use 6 different FX options in individual netting sets. These FX options are all referencing the EUR/USD exchange rate and have a maturity of 1 year, but differ with respect to option type (call/put) and moneyness (ITM/OTM/ATM). ${ }^{40}$ When calculating the exposure under the modified SA-CCR, we assume a constant market value $\left(\hat{V}_{i}(t)=V_{i}\left(t_{0}\right)\right)$ as well as a constant exchange rate $\left(\hat{P}_{i}(t)=P_{i}\left(t_{0}\right)\right)$. This is a simplification in line with the methodology presented in Sect. 3.2.3. The option volatility used for the calculation of the option price at $\left(t=t_{0}\right)$ and $\delta_{i}(t)$ is calibrated based on FX option strategies as described in Sect. 4.

Table 6 provides an overview on the CVA results for the different options. The results for ATM and ITM options are reasonable and well in line with the results from the BMM $(-2126,-2128,-2130,-2133)$. With respect to OTM options, the modified SA-CCR does not meet the results produced by the BMM. This is consistent with the outcome for FX forwards and results form the fact that we are calibrating the modified SA-CCR to ATM options. Hence, we are not able to cover the FX volatility skew in the modified SA-CCR. This leads to an underestimation of exposure for OTM FX options. An improvement of the calibration to capture the volatility surface as a whole should significantly increase the accuracy for OTM options.

\subsection{Implications for the supervisory SA-CCR}

When deriving our modified approach, we are keeping key building blocks and elements of the supervisory SA-CCR. The EaD calculated under the superviory SA-CCR will serve as input for the calculation of other regulatory measures, such as leverage

\footnotetext{
40 The ITM call is defined setting the price/strike ratio to 1.2 , while the OTM call is constructed with a price/strike ratio of 0.8 . The put options are defined vice versa.
} 
ratio (BCBS 2014a), large exposure framework (BCBS 2014d) and the CVA risk capital charge (BCBS 2017). Hence, all banks will have to implement the SA-CCR irrespectively of the application of an internal model. Furthermore, there is ongoing discussion to limit the benefit from the application of internal models by introducing a capital floor based on the outcome of regulatory standardized approaches (BCBS 2017). Given the broad application of the SA-CCR and its subsequent impact on various regulatory measures, we are certainly justified in saying that the SA-CCR is of significant importance for the regulatory framework as a whole. Hence, systemic misjudgement of risk by the supervisory SA-CCR is not an isolated issue, but will propagate through other regulatory measures.

Our results imply that the risk sensitivity of the supervisory SA-CCR can be significantly improved by adjustments to its methodological framework and its calibration. First, the multiplier formula of the supervisory SA-CCR is very conservative as it involves a floor and uses a more conservative function than analytically implied. This leads to an insufficient recognition of risk-mitigating effects from overcollateralization (especially with respect to initial margin). Abolishing the supervisory floor and adjusting the multiplier formula would significantly improve risk sensitivity for a multitude of portfolios, especially in light of new margin requirements for OTC derivatives. Second, the lack of granularity of the supervisory factor and volatility leads to a lack of risk sensitivity. IR and FX transactions with different currency are all treated with a single risk-weight. Our results show, that the calibration of the approach for each currency has strong benefits with respect to its risk sensitivity. From our point of view the calibration of the supervisory approach should be more granular to account for the characteristics of different risk factors. Third, our results reveal that the aggregation procedures are a critical issue with respect to the accuracy of results for multi-transaction netting sets. Especially netting sets with IR or FX transactions referencing different currencies are sensitive to an insufficient consideration of those effects. Nevertheless, the supervisory SA-CCR framework is flexible enough to include more complex aggregation approaches. Hence, we strongly recommend to review the supervisory aggregation procedures and consider other forms of aggregation for IR and FX add-ons.

\section{Conclusion}

The calculation of CVA based on expected exposure profiles is crucial for financial institutions involved in derivative trading activities. As a lot of market participants are not capable of implementing and maintaining an advanced model for the estimation of the expected exposure, there is a practical need for simple, semi-analytical approaches. Existing semi-analytical approaches are often designed to reflect the properties of a specific asset class and fail to provide a holistic approach across asset classes. Furthermore, they are usually not capable of capturing effects from collateralization and margining.

This paper proposes a modified supervisory approach based on the Standardized Approach for Measuring Counterparty Credit Risk Exposures (SA-CCR). We derive necessary adjustments to the supervisory methodology and calibration, obtaining an 
approach which is applicable for the calculation of CVA for accounting and pricing purposes. Main adjustments to the supervisory SA-CCR are the change of target measure and risk-neutral calibration to market-implied volatilities. While deriving our approach, we maintain key building blocks of the supervisory approach.

Our results indicate that the modified SA-CCR is able to capture main exposure dynamics on single-transaction level for the most important asset classes (FX and IR). The risk-neutral calibration results in a reasonable level of exposure. Based on the resulting expected exposure profiles, we provide an approximation of CVA, which is close to the results produced by an advanced model. By analysing multi-transaction netting sets, we reveal that in some cases appropriate aggregation mechanisms are essential to provide a reasonable approximation of the expected exposure profile. Nevertheless, the structure of the SA-CCR allows the inclusion of additional adjustments to the aggregation of results for hedging sets and asset classes. Additional analysis regarding the application of the modified SA-CCR to further asset classes and more complex products is subject for future work.

The modified SA-CCR offers a holistic and consistent framework for the calculation of exposure profiles. All asset classes and product types are modelled based on common methodological foundations. The consistent treatment and aggregation of exposure from different product types and asset classes allows the recognition of risk-mitigating effects, such as collateralization and margining, on portfolio level. Our approach is capable of estimating the impact from margin parameters and collateral on the exposure profile. Given the high amount of flexibility with respect to the treatment of products on transaction level and the aggregation of add-ons, it is possible to add additional complexity, where needed. By keeping the key building blocks and structure of the supervisory approach, institutions have the possibility to leverage on the implementation of the supervisory SA-CCR, when applying the modified approach. Based on the empirical results presented in this paper and the high importance of accuracy in CVA calculations, we do not consider the modified SA-CCR as replacement for existing advanced models. Nevertheless, our approach is suitable for the calculation of exposure profiles for products not covered by advanced methods and offers an alternative approach for institutions not capable of maintaining own advanced models. Taking everything into consideration, we believe that our approach is of significant practical relevance and offers improvements to current industry practices with respect to the calculation of CVA outside advanced models.

\section{Acknowledgements Open Access funding provided by Projekt DEAL.}

Open Access This article is licensed under a Creative Commons Attribution 4.0 International License, which permits use, sharing, adaptation, distribution and reproduction in any medium or format, as long as you give appropriate credit to the original author(s) and the source, provide a link to the Creative Commons licence, and indicate if changes were made. The images or other third party material in this article are included in the article's Creative Commons licence, unless indicated otherwise in a credit line to the material. If material is not included in the article's Creative Commons licence and your intended use is not permitted by statutory regulation or exceeds the permitted use, you will need to obtain permission directly from the copyright holder. To view a copy of this licence, visit http://creativecommons.org/licenses/by/4.0/. 


\section{A Analytical derivation of SA-CCR model foundations}

\section{A.1 Expected exposure}

Based on the assumptions introduced by BCBS (2014b), a transaction's market value follows an arithmetic Brownian motion. Hence, the market value $(V)$ of a transcation $(i)$ at a specific future point in time $(t)$ is generally defined as:

$$
V_{i}(t)=V_{i}\left(t_{0}\right)+\mu_{i} \cdot d t+\sigma_{i}(t) \cdot \sqrt{t} \cdot X_{i}
$$

where $X_{i}$ is a standard normal random variable $\left(X_{i} \sim N(0,1)\right) . \mu_{i}$ equals the drift and $\sigma_{i}(t)$ the time-dependent volatility of the transaction's market value. The SA-CCR add-on is calculated under the assumption of zero drift $\left(\mu_{i}=0\right)$, an initial market value of $0\left(V_{i}\left(t_{0}\right)=0\right)$ and absence of collateral. Furthermore, it is assumed that there are no cashflows between $t_{0}$ and $t$. Based on these assumptions, Eq. (A.1) reduces to:

$$
V_{i}(t)=1_{\left\{M_{i} \geq t\right\}} \cdot \sigma_{i}(t) \cdot \sqrt{t} \cdot X_{i}
$$

where $1_{\{\cdot\}}$ represents an indicator variable, which recognizes if the transaction has matured. $\sigma_{i}$ represents the volatility of the transaction's market value. Hence, for $M_{i} \geq t$ the market value of the transaction at time $t$ is also normally distributed with $V_{i}(t) \sim N\left(0, \sigma_{i}(t)^{2} \cdot t\right)$.

Based on Eq. (A.2) and the aforementioned assumptions, the market value of a netting set, representing a group of legally nettable transactions, is defined as the sum of the single market values of all transactions $(i)$ being an element of netting set $k$ :

$$
V_{k}(t)=\sum_{i \in k} V_{i}(t)=\sum_{i \in k} 1_{\left\{M_{i} \geq t\right\}} \cdot \sigma_{i}(t) \cdot \sqrt{t} \cdot X_{i}
$$

As the sum of joint normally distributed random variables is again normally distributed, the market value of the netting set at time $t$ is normally distributed. This results from the assumption that $X_{i}$ is a standard normal random variable. Hence, under the assumption of fixed correlations between the market value of a netting set's transactions, the variance of the $V_{k}(t)$ can be calculated the following way: ${ }^{41}$

$$
\begin{aligned}
\operatorname{Var}\left(V_{k}(t)\right) & =\operatorname{Var}\left(\sum_{i \in k} V_{i}(t)\right) \\
& =\operatorname{Var}\left(\sum_{i \in k} \sigma_{i}(t) \cdot \sqrt{t} \cdot X_{i} \cdot 1_{\left\{M_{i} \geq t\right\}}\right) \\
& =t \cdot\left(\sum_{i, j} \sigma_{i}(t) \cdot \sigma_{j}(t) \cdot \operatorname{COV}\left(X_{i}, X_{j}\right) \cdot 1_{\left\{M_{i} \geq t\right\}} \cdot 1_{\left\{M_{j} \geq t\right\}}\right)
\end{aligned}
$$

\footnotetext{
41 The assumption of fixed correlations implies that correlations between the market value of transactions in the netting set are not a function of time.
} 


$$
\begin{aligned}
& =t \cdot\left(\sum_{i, j} \sigma_{i}(t) \cdot \sigma_{j}(t) \cdot \rho_{i j} \cdot 1_{\left\{M_{i} \geq t\right\}} \cdot 1_{\left\{M_{j} \geq t\right\}}\right) \\
& =: t \cdot\left(\sigma_{k}(t)\right)^{2}
\end{aligned}
$$

Based on this result, $V_{k}(t)$ is normally distributed with $N \sim\left(0, t \cdot\left(\sigma_{k}(t)\right)^{2}\right)$. In case of a known correlation $\rho_{i j}$ between two normal random variables $X_{i}$ and $X_{j}$, equation A. 3 can be restated as follows:

$$
V_{k}(t)=\sigma_{k}(t) \cdot \sqrt{t} \cdot Y
$$

where $Y \sim N(0,1)$ and $\sigma_{k}(t)$ represents the volatility of the netting set's market value:

$$
\sigma_{k}(t)=\sqrt{\sum_{i, j} \rho_{i j} \cdot \sigma_{i}(t) \cdot \sigma_{j}(t) \cdot 1_{\left\{M_{i} \geq t\right\}} \cdot 1_{\left\{M_{j} \geq t\right\}}}
$$

Please note that the netting set's volatility $\sigma_{k}(t)$ is itself a function of $t$. This dependence results from the changing composition of the netting set, as transactions mature over time as well as changes in the volatility of the transactions' market values. The target measure $\left(E E_{k}(t)\right)$ is formally defined as the expected positive value of the netting set's market value. Hence, we are able to calculate the Expected Exposure of a netting set by:

$$
E E_{k}(t)=\mathbb{E}^{Q}\left[\max \left(V_{k}(t), 0\right)\right]=\mathbb{E}^{Q}\left[\max \left(\sigma_{k}(t) \cdot \sqrt{t} \cdot Y, 0\right)\right]
$$

As $Y$ is a standard normal variable, we are able to calculate the expected value of $Y$ and hence $E E(t)$ analytically:

$$
\begin{aligned}
\operatorname{Add} O n_{k}=E E(t) & =\mathbb{E}^{Q}\left[\max \left(V_{k}(t), 0\right)\right]=\mathbb{E}^{Q}\left[\max \left(\sigma_{k}(t) \cdot \sqrt{t} \cdot Y, 0\right)\right] \\
& =\sigma_{k}(t) \cdot \sqrt{t} \cdot \mathbb{E}^{Q}[\max (Y, 0)] \\
& =\sigma_{k}(t) \cdot \sqrt{t} \cdot \int_{0}^{+\infty} \frac{1}{\sqrt{2 \pi}} \cdot \exp \left[\frac{-y^{2}}{2}\right] y d y \\
& =\sigma_{k}(t) \cdot \sqrt{t} \cdot \frac{1}{\sqrt{2 \pi}} \cdot\left[-e^{\frac{-y^{2}}{2}} d y\right]_{0}^{+\infty} \\
& =\sigma_{k}(t) \cdot \sqrt{t} \cdot \frac{1}{\sqrt{2 \pi}} \cdot[-(0-1)] \\
& =\sigma_{k}(t) \cdot \sqrt{t} \cdot \frac{1}{\sqrt{2 \pi}} \\
& =\sigma_{k}(t) \cdot \sqrt{t} \cdot \phi(0)
\end{aligned}
$$

where $\phi(0)$ is defined as the standard normal probability density: $\phi(0)=1 / \sqrt{2 \pi}$. 


\section{A. 2 Aggregating trade level add-ons}

The regulatory and modified SA-CCR are aiming for calculation of aggregated addons instead of dealing with trade-level volatilities directly. Hence, Eq. (A.8) needs to be restated the following way:

$$
E E_{k}(t)=A d d O n_{k}(t)=\sqrt{\sum_{i, j} \rho_{i j} \cdot \operatorname{AddOn_{i}}(t) \cdot \operatorname{AddOn_{j}(t)}}
$$

where $\mathrm{AddOn}_{i}(t)$ represents the expected exposure of a netting set with one trade $(i)$ at $t$ :

$$
A d d O n_{i}(t)=E E_{i}(t)=\sigma_{i}(t) \cdot \sqrt{t} \cdot \phi(0)
$$

By inserting Eq. (A.10) into Eq. (A.9), we are able to show that trade level add-ons can be aggregated as if they were standard deviations:

$$
\begin{aligned}
\operatorname{AddOn}_{k}(t) & =\sqrt{\sum_{i, j} \rho_{i j} \cdot \operatorname{AddOn}_{i}(t) \cdot \operatorname{AddOn}_{j}(t)} \\
& =\sqrt{\sum_{i, j} \rho_{i j} \cdot \sigma_{i}(t) \cdot \sqrt{t} \cdot \phi(0) \cdot \sigma_{j}(t) \cdot \sqrt{t} \cdot \phi(0)} \\
& =\sqrt{\sum_{i, j} \rho_{i j} \cdot \sigma_{i}(t) \cdot \sigma_{j}(t) \cdot t \cdot(\phi(0))^{2}} \\
& =\phi(0) \cdot \sqrt{t} \cdot \sqrt{\sum_{i, j} \rho_{i j} \cdot \sigma_{i}(t) \cdot \sigma_{j}(t)} \\
& =\phi(0) \cdot \sqrt{t} \cdot \sigma_{k}(t)
\end{aligned}
$$

Equation (A.11) is equal to the definition of the expected exposure at netting set level $E E_{k}(t)$ in Eq. (A.8). Hence, trade-level add-ons can be aggregated at each point in time $(t)$ as if they were standard deviations. This is the central foundation with respect to the aggregation procedures formulated by BCBS (2014b). As shown above, this foundation is still valid after switching to the new target measure $\left(E E_{k}(t)\right)$ for the modified SA-CCR.

\section{A.3 PFE multiplier formulation}

\section{A.3.1 Multiplier formula for unmargined netting sets}

As discussed in Sect. 3.3, the expected exposure $(E E(t))$ of an unmargined netting set $(k)$ at time $t$ under the presence of collateral and a market value different from zero is defined as follows: 


$$
E E_{k}(t)=\mathbb{E}^{Q}\left[\max \left(\hat{V}_{k}(t)+\sigma_{k}(t) \cdot \sqrt{t} \cdot Y-C_{C E}(t), 0\right)\right]
$$

where

- $\hat{V}_{k}(t)$ is the (deterministic) market value of the netting set at time $t$,

- $C_{C E}(t)$ equals the cash-equivalent collateral value at time $t$ as defined by Eq. (10)

$-\sigma_{k}(t)$ is the volatility of the netting set at time $t$,

$-Y$ is defined as a standard normal random variable.

For the derivation of the multiplier formula, the following definitions are applied:

$$
\begin{array}{r}
X:=\hat{V}_{k}(t)+\sigma_{k}(t) \cdot \sqrt{t} \cdot Y-C_{C E}(t) \\
\mathbb{E}[X]=\hat{V}_{k}(t)-C_{C E}(t)=: x_{0} \\
\operatorname{Var}[X]=\left(\sigma_{k}(t)\right)^{2} \cdot t=: \sigma^{2}
\end{array}
$$

These definitions imply that $X$ is normally distributed with $X \sim N\left(x_{0}, \sigma^{2}\right)$. Hence, the Expected Exposure $\left(E E_{k}(t)\right)$ can be calculated analytically by solving:

$$
E E_{k}(t)=\mathbb{E}^{Q}[\max (X, 0)]
$$

Applying the probability density function of a normally distributed random variable leads to the following formulation for $E E_{k}(t)$ :

$$
E E_{k}(t)=\int_{0}^{\infty} x \cdot \frac{1}{\sigma \cdot \sqrt{2 \cdot \pi}} \cdot \exp \left(-\frac{\left(x-x_{0}\right)^{2}}{2 \sigma^{2}}\right) d x
$$

In order to solve Eq. (A.17), the following substitutions have to be applied: $y=\frac{x-x_{0}}{\sigma}$ and $\sigma \cdot d y=d x$.

$$
E E_{k}(t)=\frac{1}{\sqrt{2 \pi}} \int_{\frac{-x_{0}}{\sigma}}^{\infty}\left(x_{0}+\sigma \cdot y\right) \cdot \exp \left(-\frac{y^{2}}{2}\right) d y
$$

Equation (A.18) can be solved analytically:

$$
\begin{aligned}
E E_{k}(t) & =\frac{1}{\sqrt{2 \pi}} \int_{\frac{-x_{0}}{\sigma}}^{\infty}\left(x_{0}+\sigma \cdot y\right) \cdot \exp \left(-\frac{y^{2}}{2}\right) d y \\
& =x_{0} \cdot \int_{\frac{-x_{0}}{\sigma}}^{\infty} \frac{1}{\sqrt{2 \pi}} \exp \left(-\frac{y^{2}}{2}\right) d y+\sigma \cdot \int_{\frac{-x_{0}}{\sigma}}^{\infty} \frac{1}{\sqrt{2 \pi}} \cdot y \cdot \exp \left(-\frac{y^{2}}{2}\right) d y \\
& =x_{0} \cdot[\Phi(y)]_{\frac{-x_{0}}{\sigma}}^{\infty}+\sigma \cdot\left[-\frac{1}{\sqrt{2 \pi}} \cdot \exp \left(-\frac{y^{2}}{2}\right)\right]_{\frac{-x_{0}}{\sigma}}^{\infty} \\
& =x_{0} \cdot\left[1-\Phi\left(-\frac{x_{0}}{\sigma}\right)\right]+\sigma \cdot\left[-0+\frac{1}{\sqrt{2 \pi}} \cdot \exp \left(\frac{-x_{0}^{2}}{2 \sigma^{2}}\right)\right] \\
& =x_{0} \cdot \Phi\left(\frac{x_{0}}{\sigma}\right)+\sigma \cdot \phi\left(\frac{x_{0}}{\sigma}\right)
\end{aligned}
$$


After replacing $x_{0}$ and $\sigma$ with the values defined in equations (A.14) and (A.15) we arrive at:

$$
\begin{aligned}
E E_{k}(t)= & {\left[\hat{V}_{k}(t)-C_{C E}(t)\right] \cdot \Phi\left(\frac{\hat{V}_{k}(t)-C_{C E}(t)}{\sigma_{k}(t) \cdot \sqrt{t}}\right) } \\
& +\sigma_{k}(t) \cdot \sqrt{t} \cdot \phi\left(\frac{\hat{V}_{k}(t)-C_{C E}(t)}{\sigma_{k}(t) \cdot \sqrt{t}}\right)
\end{aligned}
$$

where $\Phi(\cdot)$ is the standard normal cumulative distribution function and $\phi(\cdot)$ the standard normal probability density function. This equation is equivalent to the result for calculating the $\mathrm{EE}(\mathrm{t})$ for unmargined netting sets according to equation (41) in $\operatorname{BCBS}(2014 \mathrm{~b})$. By assuming $\left[\hat{V}_{k}(t)-C_{C E}(t)\right]=0$, we receive $E E(t)$ equal to the formulation of Eq. (20):

$$
A d d O n_{k}(t)=E E_{k}(t)=\sigma_{k}(t) \cdot \sqrt{t} \cdot \phi(0)
$$

As we want to express the multiplier in terms of $A d d O n_{k}(t)$ rather than netting set volatilities, we solve Eq. (A.21) for $\sigma_{k}(t)$. This results in:

$$
\sigma_{k}(t)=\frac{\operatorname{Add} O n_{k}(t)}{\sqrt{t} \cdot \phi(0)}
$$

Inserting Eq. (A.22) into Eq. (A.20) leads to:

$$
\begin{aligned}
E E_{k}(t)= & {\left[\hat{V}_{k}(t)-C_{C E}(t)\right] \cdot \Phi\left(\phi(0) \cdot \frac{\hat{V}_{k}(t)-C_{C E}(t)}{A d d O n_{k}(t)}\right) } \\
& +\frac{A d d O n_{k}(t)}{\phi(0)} \cdot \phi\left(\phi(0) \cdot \frac{\hat{V}_{k}(t)-C_{C E}(t)}{A d d O n_{k}(t)}\right)
\end{aligned}
$$

Based on Eq. (A.23), we are able to derive the multiplier formula in accordance with BCBS (2014b) by isolating the PFE portion of Eq. (A.23). Therefore, we need to subtract the replacement costs from $E E_{k}(t)$ :

$$
\begin{aligned}
& P F E_{k}(t)=\left[\hat{V}_{k}(t)-C_{C E}(t)\right] \cdot \Phi\left(\phi(0) \cdot \frac{\hat{V}_{k}(t)-C_{C E}(t)}{\left.\operatorname{AddOn_{k}(t)}\right)}\right. \\
& +\frac{A d d O n_{k}(t)}{\phi(0)} \cdot \phi\left(\phi(0) \cdot \frac{\hat{V}_{k}(t)-C_{C E}(t)}{A d d O n_{k}(t)}\right)-R C_{k}(t)
\end{aligned}
$$

Based on Eq. (18), the multiplier of a netting set at time $\mathrm{t}\left(m_{k}(t)\right)$ is defined as the ratio of potential future exposure and $A d d O n_{k}(t)$ :

$$
m_{k}(t)=\frac{P F E_{k}(t)}{\operatorname{AddOn_{k}(t)}}
$$


Hence, we arrive at the the following formula for the multiplier:

$$
\begin{aligned}
& m_{k}(t)=\frac{\left[\hat{V}_{k}(t)-C_{C E}(t)\right]}{A d d O n_{k}(t)} \cdot \Phi\left(\phi(0) \cdot \frac{\hat{V}_{k}(t)-C_{C E}(t)}{A d d O n_{k}(t)}\right) \\
& +\frac{1}{\phi(0)} \cdot \phi\left(\phi(0) \cdot \frac{\hat{V}_{k}(t)-C_{C E}(t)}{A d d O n_{k}(t)}\right)-\frac{\max \left(\hat{V}_{k}(t)-C_{C E}(t), 0\right)}{A d d O n_{k}(t)}
\end{aligned}
$$

By substituting $\frac{\hat{V}_{k}(t)-C_{C E}(t)}{A d d O n_{k}(t)}=y$ we arrive at the following final formulation for the multiplier:

$$
m_{k}(t)=y \cdot \Phi[\phi(0) y]+\frac{\phi[\phi(0) y]}{\phi(0)}-\frac{\max \left(\hat{V}_{k}(t)-C_{C E}(t), 0\right)}{\operatorname{AddOn_{k}(t)}}
$$

\section{A.3.2 Multiplier formula for margined netting sets}

Based on assumptions presented in Sect. 3.3, the expected exposure of a margined netting set is defined as:

$$
E E_{k}(t)=\mathbb{E}^{Q}\left[\max \left(\hat{V}_{k}(t)+\sigma_{k}(t) \cdot \sqrt{M P O R} \cdot Y-\hat{C}_{C E}(t), 0\right)\right]
$$

where:

- $\hat{V}_{k}(t)$ is the (deterministic) market value of the netting set at time $t$,

- $\hat{C}_{C E}(t)$ represents the (deterministic) cash-equivalent collateral value at $t$ as defined by Eq. (17),

$-\sigma_{k}(t)$ is the volatility of the netting set at time $t$,

$-Y$ is defined as a standard normal random variable.

Based on Eq. (A.28) the following definitions are applied:

$$
\begin{array}{r}
X:=\hat{V}_{k}(t)+\sigma_{k}(t) \cdot \sqrt{M P O R} \cdot Y-\hat{C}_{C E}(t) \\
\mathbb{E}[X]=\hat{V}_{k}(t)-\hat{C}_{C E}(t)=: x_{0} \\
\operatorname{Var}[X]=\left(\sigma_{k}(t)\right)^{2} \cdot(\sqrt{M P O R})^{2}=: \sigma^{2}
\end{array}
$$

These definitions imply that $X$ is normally distributed with $X \sim N\left(x_{0}, \sigma^{2}\right)$. Hence, the Expected Exposure $\left(E E_{k}(t)\right)$ can be calculated analytically by solving:

$$
E E_{k}(t)=\mathbb{E}^{Q}[\max (X, 0)]
$$

Based on this formulation, we are able to solve the equation analytically by applying the same steps as presented in "Appendix A.3.1". Hence, we arrive at the following formulation for the multiplier: 


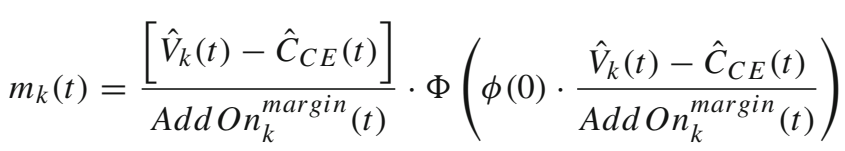

$$
\begin{aligned}
& +\frac{1}{\phi(0)} \cdot \phi\left(\phi(0) \cdot \frac{\hat{V}_{k}(t)-\hat{C}_{C E}(t)}{A d d O n_{k}^{\text {margin }}(t)}\right)-\frac{\max \left(\hat{V}_{k}(t)-\hat{C}_{C E}(t), 0\right)}{A d d O n_{k}^{\text {margined }}(t)}
\end{aligned}
$$

By substituting $\frac{\hat{V}_{k}(t)-\hat{C}_{C E}(t)}{A d d O n_{k}^{\text {margin }}(t)}=y$ we arrive at the following formula for the multiplier:

$$
m_{k}(t)=y \cdot \Phi[\phi(0) y]+\frac{\phi[\phi(0) y]}{\phi(0)}-\frac{\max \left(\hat{V}_{k}(t)-\hat{C}_{C E}(t), 0\right)}{A d d O n_{k}^{\text {margined }}(t)}
$$

\section{A.3.3 Adjusted notional calculation by asset class}

In general, we follow the asset class specific calculation procedures for the determination of the adjusted notional amount $\left(d_{i}(t)\right)$ introduced by BCBS $(2014 \mathrm{~b})$. Hence, the following paragraphs do not provide a detailed discussion of the asset class specific features of the SA-CCR, but concentrate on elements that have been modified when developing our approach.

For the derivation of the adjusted notional for interest rate (IR) swaps, we allow cash flows during the life-time of interest rate derivatives. The main purpose of the add-on calculation for IR transactions within the modified SA-CCR is to capture the diffusion and the amortization effect. BCBS (2014b) derives the calculation of the adjusted notional for interest rate derivatives based on the following pricing formula for a fixed-to-floating swap:

$$
V_{i}^{\text {swap }}(t)=\left[S R_{i}(t)-F R_{i}\right] \cdot \int_{\max \left(S_{i}, t\right)}^{E_{i}} N_{i}(\tau) \cdot D F(t, \tau) d \tau
$$

where $S R_{i}(t)$ equals the swap rate at time $t$ and $F R_{i}$ is the fixed rate of the swap. $D F(t, \tau)$ represents the discount factor from time $\tau$ to time $t . E_{i}$ represents the time until the end date of the transaction at time $t$ while $S_{i}$ equals the time until the start date of the transaction at time $t$ (in years). The volatility of the swap rate describes the diffusion effect of the exposure and is captured by the exposure factor $\left(E F_{i}\right)$ in Eq. (25). Thus the adjusted notional parameter is meant to provide an approximation for the integral at time $t$. A deviation from the supervisory approach is introduced. Instead of calculating the value under the integral at $t=t_{0}$ or the start date of the swap respectively, we estimate the value at time $\left(t^{*}\right)$ :

$$
d_{i}^{(I R)}(t)=\bar{N}_{i} \cdot D_{i}(t)=\bar{N}_{i} \int_{t^{*}}^{E_{i}} D F(t, \tau) d \tau
$$


where $t^{*}$ is defined as the maximum of $t$ and the start date of the interest rate $\operatorname{swap}\left(S_{i}\right)$. $\bar{N}_{i}$ is the average of the swap notional between $t$ and the end date of the transaction $\left(E_{i}\right) . D_{i}(t)$ represents the approximation of the integral under the assumption of a flat interest rate curve. $D_{i}(t)$ is a measure for the duration of the swap and solves for:

$$
D_{i}(t)=\int_{t^{*}}^{E_{i}} \exp (-r \tau) d \tau=\frac{\exp \left(-r \cdot t^{*}\right)-\exp \left(-r \cdot E_{i}\right)}{r}
$$

With increasing $t, D_{i}(t)$ decreases. This behaviour captures the amortization effect of interest rates swaps. In case of a cash-flow based calculation of the expected future market value for replacement costs (see Eq. (4)) the duration parameter $\left(D_{i}(t)\right)$ is replaced by the actual (absolute) duration of the swap position.

In accordance with BCBS (2014b), Eq. (A.37) is also applied to credit derivatives. Hence, the adjusted notional amount for credit and interest rate derivatives is a function of time and defined as:

$$
d_{i}^{(I R / C R)}(t)=\bar{N}_{i} \cdot D_{i}(t)=\bar{N}_{i} \cdot \frac{\exp \left(-r \cdot t^{*}\right)-\exp \left(-r \cdot E_{i}\right)}{r}
$$

For FX derivatives the adjusted notional amount is defined as the notional of the foreign currency leg denominated in the reporting / domestic currency $(D)$ :

$$
d_{i}^{(F X)}(t)=\bar{N}_{i}^{\left(A_{i}\right)} \cdot \hat{P}_{\frac{A_{i}}{D}}(t)
$$

where $A_{i}$ represents the foreign currency of the transaction and $D$ the reporting currency. $P_{\frac{A_{i}}{D}}$ equals the price of one unit foreign currency in domestic currency. As $P_{\frac{A_{i}}{D}}$ is not known for future points in time, an estimation of $P_{\frac{A_{i}}{D}}(t)$ is required. For FX products, we use the respective forward exchange rate for determination of the adjusted notional amount at time $t$ :

$$
\hat{P}_{\frac{A_{i}}{D}}(t)=F_{\frac{A_{i}}{D}}\left(t_{0}, t\right)=P_{\frac{A_{i}}{D}}\left(t_{0}\right) \cdot e^{\left(r_{D}-r_{A_{i}}\right) \cdot t}
$$

If both legs of the FX product are denominated in foreign currency $\left(A_{i}\right.$ and $\left.B_{i}\right), d_{i}$ is calculated as follows:

$$
d_{i}^{(F X)}(t)=\left(\bar{N}_{i}^{\left(A_{i}\right)} \cdot \hat{P}_{\frac{A_{i}}{D}}(t)+\bar{N}_{i}^{\left(B_{i}\right)} \cdot \hat{P}_{\frac{B_{i}}{D}}(t)\right) \cdot 0.5
$$

In line with BCBS (2014c) the adjusted notional amount for equity and commodity derivatives is defined as product of the number of units referenced by the transaction $\left(\eta_{i}\right)$ and the price of one unit $\left(P_{i}\right)$. In accordance with our approach for FX derivatives, we use the respective forward price for future dates as estimation for the price of one unit at time $t$.

$$
d_{i}^{(E Q / F X)}(t)=\eta_{i} \cdot \hat{P}_{i}(t)
$$


If there are deterministic changes in the notional of a transaction over time (e.g. amortizing interest rate swap), the future notional amount at $t$ is known. Our approach offers the flexibility to include a product specific notional profile $\left(N_{i}(t)\right)$ into the calculation of the adjusted notional.

\section{A.4 Empirical analysis}

\section{A.4.1 Calibration of the benchmark model (BMM)}

In order to benchmark the results of the modified SA-CCR, we calculate exposure profiles based on an advanced exposure model. The model involves stochastic processes for the simulation of the development of risk factors over time. The parameters of these stochastic processes have to be calibrated. In case of generation of exposure profiles for CVA calculation, the calibration is performed under the risk-neutral framework based on observable market prices for specific financial instruments. The aim of the calibration process is to find the set of model parameters $(\Omega)$ that minimizes the deviation between the prices of calibration instruments $(n)$ calculated by the model $\left(P_{n}^{\text {model }}\right)$ and their market price $\left(P_{n}^{\text {market }}\right)$ :

$$
r^{2}(\Omega)=\sum_{n=1}^{N}\left(P_{n}^{\text {market }}-P_{n}^{\text {model }}(\Omega)\right)^{2}
$$

Hence, the main inputs of the calibration process are financial instruments with observable market prices as well as their properties (such as maturity, strike, underlying). Within this section we provide an overview on the instruments and data used for the calibration of the benchmark model. However, we do not provide a detailed information on the models and the corresponding pricing functions. ${ }^{42}$

Trolle-Schwartz model: For the simulation of interest rates, we apply a model based on Trolle and Schwartz (2009). The model simulates the evolution of instantaneous forward rates with stochastic volatility. We use an extended form of the model considering a multi-curve setting under the assumptions of deterministic tenor-basis spreads. The calibration of the model parameters for each currency is based on European swaptions. Available swaption data has the following main dimensions and values:

- Option Tenor: 1M, 3M, 6M, 9M, 1Y, 2Y, 5Y, 10Y, 15Y, 20Y, 30Y

- Swap Tenor: 1Y, 2Y, 5Y, 10Y, 15Y, 20Y, 30Y

- Strike (ATM \pm bp): 12.5, 25, 50, 75, 100, 150, 200, 300

The prices of these swaptions are not directly observable in the market, as swaptions are quoted in terms of implied volatility. Hence, the observable implied volatilities have to be transformed into swaption prices. Depending on the volatility definition (lognormal/normal) we use a Black or Bachelier pricing formula to arrive at the swaption prices. These prices are used to calibrate the parameters of the Trolle-Schwartz model. Based on the available input data, we are able to recognize dependence of the volatility on the strike, underlying and option tenor.

\footnotetext{
42 Please refer to Heston (1993) and Trolle and Schwartz (2009) for additional information.
} 
Heston model: The exchange rate is simulated via a stochastic model based on Heston (1993). The exchange rate equals the price of one unit foreign currency, expressed in domestic currency. The parameters for modelling the interest rate process of the foreign and domestic currency are given by the calibration of the Trolle-Schwartz model. The other parameters of the model are calibrated based on European options on exchange rates. There are no quotes for FX options directly available in the market. Quotes are only directly observable for FX option strategies in the form of lognormal volatilities as function of delta. Available FX option strategy data for each exchange rate has the following main dimensions and values:

- Strategy type: Risk Reversal, Straddle, Strangle

- Maturity: 1D, 1W, 2W, 3W, 1M, 2M, 3M, 6M, 9M, 1Y, 2Y, 3Y, 4Y, 5Y, 7Y, 10Y

- Delta: $0.5,0.25,0.1$

For each maturity, each of these strategies is converted into European call and put options. This results in a set of five options for each maturity ( 3 call and 2 put options). The respective implied volatility of each option is transferred into a market price. This set of options with the corresponding market prices is used to calibrate the parameters of the Heston model. The resulting model is able to capture the dependency of the implied volatility to the moneyness and maturity.

\section{A.4.2 Input: transactions and netting sets}

See Table 7.

Table 7 Hypothetical netting sets for empirical analysis in Sect. 5

\begin{tabular}{llllll}
\hline Id & TradeId & Class & Description & Notional(EUR) & mtm(EUR) \\
\hline-2000 & -2000 & IR & EUR IRS PAY ATM-0.01 5Y & $1,000,000$ & $49,937.01$ \\
-2001 & -2001 & IR & EUR IRS PAY ATM 5Y & $1,000,000$ & -0.01 \\
-2002 & -2002 & IR & EUR IRS PAY ATM+0.01 5Y & $1,000,000$ & $-49,937.03$ \\
-2003 & -2003 & IR & EUR IRS REC ATM-0.01 5Y & $1,000,000$ & $-49,937.01$ \\
-2004 & -2004 & IR & EUR IRS REC ATM 5Y & $1,000,000$ & 0.01 \\
-2005 & -2005 & IR & EUR IRS REC ATM+0.01 5Y & $1,000,000$ & $49,937.03$ \\
-2006 & -2006 & IR & EUR IRS PAY ATM-0.01 10Y & $1,000,000$ & $97,419.36$ \\
-2007 & -2007 & IR & EUR IRS PAY ATM10Y & $1,000,000$ & -0.02 \\
-2008 & -2008 & IR & EUR IRS PAY ATM+0.01 10Y & $1,000,000$ & $-97,419.39$ \\
-2009 & -2009 & IR & EUR IRS PAY ATM-0.01 7Y & $1,000,000$ & $69,387.70$ \\
-2010 & -2010 & IR & EUR IRS PAY ATM 7Y & $1,000,000$ & 0 \\
-2011 & -2011 & IR & EUR IRS PAY ATM+0.01 7Y & $1,000,000$ & $-69,387.71$ \\
-2012 & -2012 & IR & USD IRS PAY ATM-0.01 5Y & $1,000,000$ & $46,129.85$ \\
-2013 & -2013 & IR & USD IRS PAY ATM 5Y & $1,000,000$ & 0.01 \\
-2014 & -2014 & IR & USD IRS PAY ATM+0.01 5Y & $1,000,000$ & $-46,129.83$ \\
-2015 & -2015 & FX & EUR/USD FWD ATM-20\% 1Y & $1,000,000$ & $-205,592.64$ \\
-2016 & -2016 & FX & EUR/USD FWD ATM 1Y & $1,000,000$ & 171.95 \\
\hline
\end{tabular}


Table 7 continued

\begin{tabular}{|c|c|c|c|c|c|}
\hline Id & TradeId & Class & Description & Notional(EUR) & $\operatorname{mtm}($ EUR $)$ \\
\hline-2017 & -2017 & FX & EUR/USD FWD ATM+20\% $1 \mathrm{Y}$ & $1,000,000$ & $205,936.54$ \\
\hline-2018 & -2018 & FX & EUR/USD FWD ATM-20\% 3Y & $1,000,000$ & $-217,299.87$ \\
\hline-2019 & -2019 & FX & EUR/USD FWD ATM 3Y & $1,000,000$ & 144.92 \\
\hline-2020 & -2020 & FX & EUR/USD FWD ATM+20\% $3 \mathrm{Y}$ & $1,000,000$ & $217,589.71$ \\
\hline-2021 & -2021 & $\mathrm{FX}$ & EUR/JPY FWD ATM-20\% 3Y & $1,000,000$ & $-200,390.47$ \\
\hline-2022 & -2022 & FX & EUR/JPY FWD ATM 3Y & $1,000,000$ & -16.85 \\
\hline-2023 & -2023 & FX & EUR/JPY FWD ATM+20\% 3 Y & $1,000,000$ & $200,356.78$ \\
\hline-2024 & -2024 & FX & EUR/GBP FWD ATM-20\% 3Y & $1,000,000$ & $-206,765.66$ \\
\hline-2025 & -2025 & FX & EUR/GBP FWD ATM 3Y & $1,000,000$ & 54.23 \\
\hline-2026 & -2026 & FX & EUR/GBP FWD ATM+20\% $3 \mathrm{Y}$ & $1,000,000$ & $206,874.12$ \\
\hline-2027 & -2027 & FX & EUR/CHF FWD ATM-20\% 1Y & $1,000,000$ & $-199,214.46$ \\
\hline-2028 & -2028 & FX & EUR/CHF FWD ATM 1Y & $1,000,000$ & -22.31 \\
\hline-2029 & -2029 & FX & EUR/CHF FWD ATM+20\% $1 \mathrm{Y}$ & $1,000,000$ & $199,169.84$ \\
\hline-2030 & -2030 & FX & USD/GBP FWD ATM-20\% 3Y & $1,000,000$ & $-200,050.19$ \\
\hline-2031 & -2031 & FX & USD/GBP FWD ATM 3Y & $1,000,000$ & -80.85 \\
\hline-2032 & -2032 & FX & USD/GBP FWD ATM+20\% $3 \mathrm{Y}$ & $1,000,000$ & $199,888.48$ \\
\hline \multirow[t]{2}{*}{-2033} & -2001 & IR & EUR IRS PAY ATM 5Y & $1,000,000$ & -0.01 \\
\hline & -2004 & IR & EUR IRS REC ATM $5 Y$ & $1,000,000$ & 0.01 \\
\hline \multirow[t]{3}{*}{-2034} & -2001 & IR & EUR IRS PAY ATM 5Y & $1,000,000$ & -0.01 \\
\hline & -2007 & IR & EUR IRS PAY ATM10Y & $1,000,000$ & -0.02 \\
\hline & -2010 & IR & EUR IRS PAY ATM 7Y & $1,000,000$ & 0 \\
\hline \multirow[t]{2}{*}{-2035} & -2013 & IR & USD IRS PAY ATM 5Y & $1,000,000$ & 0.01 \\
\hline & -2001 & IR & EUR IRS PAY ATM 5Y & $1,000,000$ & -0.01 \\
\hline \multirow[t]{3}{*}{-2036} & -2012 & IR & USD IRS PAY ATM-0.015Y & $1,000,000$ & $46,129.85$ \\
\hline & -2013 & IR & USD IRS PAY ATM 5Y & $1,000,000$ & 0.01 \\
\hline & -2014 & IR & USD IRS PAY ATM+0.015Y & $1,000,000$ & $-46,129.83$ \\
\hline \multirow[t]{2}{*}{-2037} & -2019 & FX & EUR/USD FWD ATM 3Y & $1,000,000$ & 144.92 \\
\hline & -2022 & FX & EUR/JPY FXFWD ATM $3 Y$ & $1,000,000$ & -16.85 \\
\hline \multirow[t]{2}{*}{-2038} & -2019 & $\mathrm{FX}$ & EUR/USD FWD ATM 3Y & $1,000,000$ & 144.92 \\
\hline & -2031 & FX & USD/GBP FWD ATM $3 Y$ & $1,000,000$ & -80.85 \\
\hline \multirow[t]{2}{*}{-2039} & -2028 & FX & EUR/CHF FWD ATM 1Y & $1,000,000$ & -22.31 \\
\hline & -2031 & FX & USD/GBP FWD ATM $3 Y$ & $1,000,000$ & -80.85 \\
\hline \multirow[t]{2}{*}{-2040} & -2019 & FX & EUR/USD FWD ATM 3 Y & $1,000,000$ & 144.92 \\
\hline & -2016 & FX & EUR/USD FWD ATM $1 Y$ & $1,000,000$ & 171.95 \\
\hline \multirow[t]{2}{*}{-2041} & -2019 & FX & EUR/USD FWD ATM $3 Y$ & $1,000,000$ & 144.92 \\
\hline & -2004 & IR & EUR IRS REC ATM 5Y & $1,000,000$ & 0.01 \\
\hline-2126 & -2126 & FX & EUR/USD FX Call Option ATM 1Y & 1.000 .000 & $29,993.39$ \\
\hline-2128 & -2128 & FX & EUR/USD FX Put Option ATM 1Y & 1.000 .000 & $29,993.39$ \\
\hline-2130 & -2130 & FX & EUR/USD FX Call Option ITM 1Y & 1.000 .000 & $200,028.82$ \\
\hline
\end{tabular}


Table 7 continued

\begin{tabular}{llllll}
\hline Id & TradeId & Class & Description & Notional(EUR) & mtm(EUR) \\
\hline-2131 & -2131 & FX & EUR/USD FX Put Option OTM 1Y & 1.000 .000 & 28.82 \\
-2132 & -2132 & FX & EUR/USD FX Call Option OTM 1Y & 1.000 .000 & 28.82 \\
-2133 & -2133 & FX & EUR/USD FX Put Option ITM 1Y & 1.000 .000 & $200,028.82$ \\
\hline
\end{tabular}

This table provides an overview of the hypothetical netting sets, which are used within the empirical analysis presented in Sect. 5 of this paper. All IR trades are fix-to-float IR swaps

\section{A.4.3 Output: IR profiles}

See Figs. 4, 5, 6, 7, and 8.
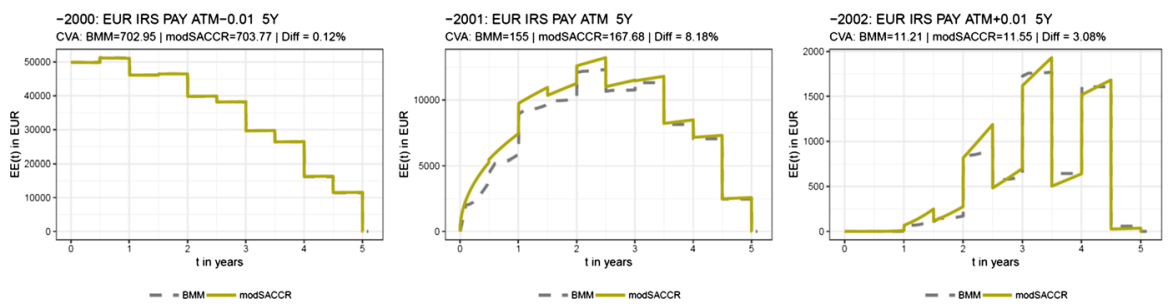

Fig. 4 Expected exposure of EUR 5Y IR payer swaps. Note The figure shows the expected exposure profile and CVA results for EUR 5Y IR payer swaps with different moneyness calculated with the modified SA-CCR (solid line) and the benchmark model (dashed line). Please notice that in case of a payer swap, ATM-0.01 (left panel) is equal to an in-the-money position
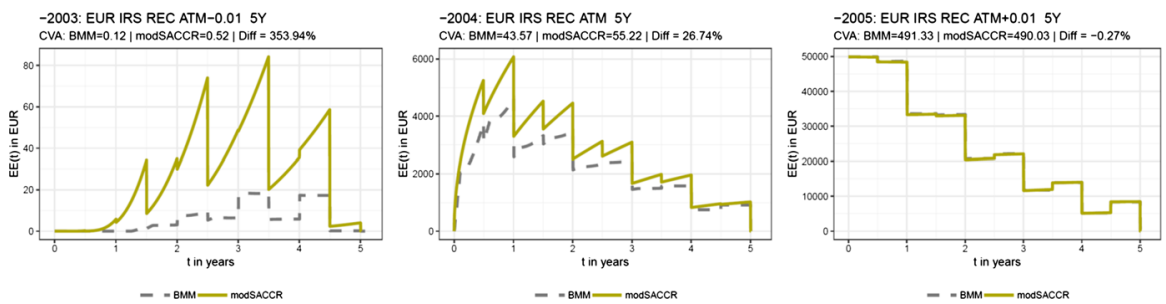

Fig. 5 Expected exposure of EUR 5Y IR receiver swaps. Note The figure shows the expected exposure profile and CVA results for EUR 5Y IR receiver swaps with different moneyness calculated with the modified SA-CCR (solid line) and the benchmark model (dashed line). Please notice that in case of a receiver swap, ATM-0.01 (left panel) is equal to an out-the-money position 

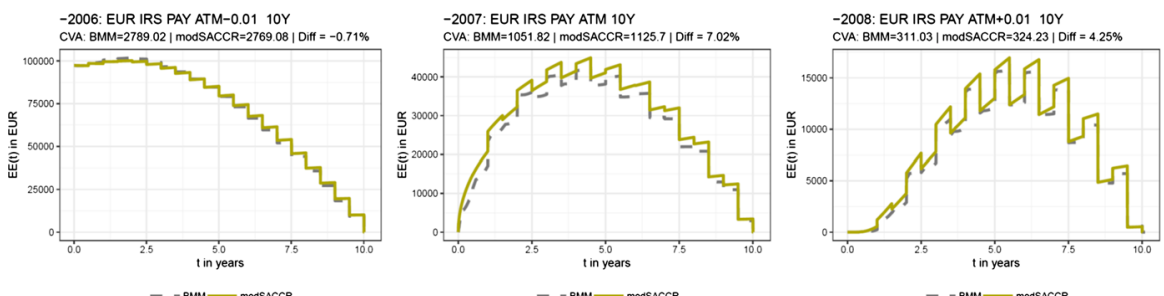

Fig. 6 Expected exposure of EUR 10 IR payer swaps. Note The figure shows the expected exposure profile and CVA results for EUR 10Y IR payer swaps with different moneyness calculated with the modified SA-CCR (solid line) and the benchmark model (dashed line)
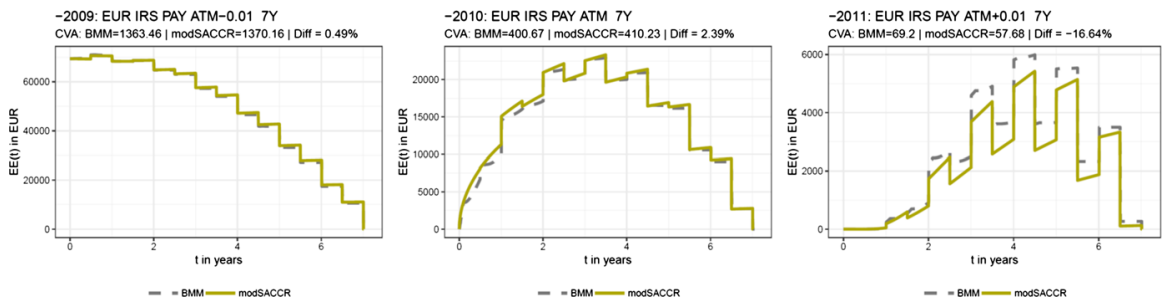

Fig. 7 Expected exposure of EUR 7Y IR payer swaps. Note The figure shows the expected exposure profile and CVA results for EUR 7Y IR payer swaps with different moneyness calculated with the modified SA-CCR (solid line) and the benchmark model (dashed line)

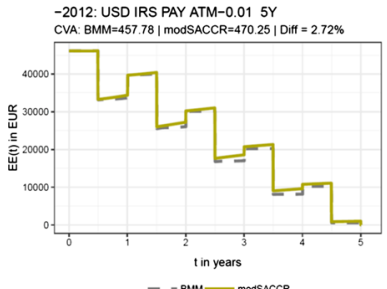

- - BMM- modSACCA

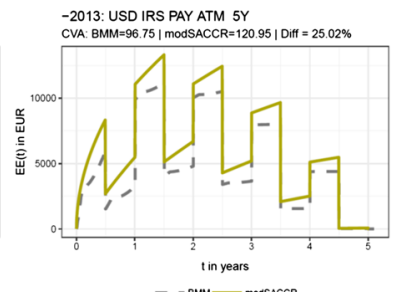

- - BMM- mOSAACCR

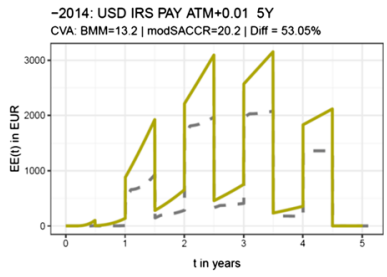

- BMm- modsacce

Fig. 8 Expected exposure of USD 5Y IR payer swaps. Note The figure shows the expected exposure profile and CVA results for USD 5Y IR payer swaps with different moneyness calculated with the modified SA-CCR (solid line) and the benchmark model (dashed line) 


\section{A.4.4 Output: FX profiles}

See Figs. 9, 10, 11, 12, 13 and 14.
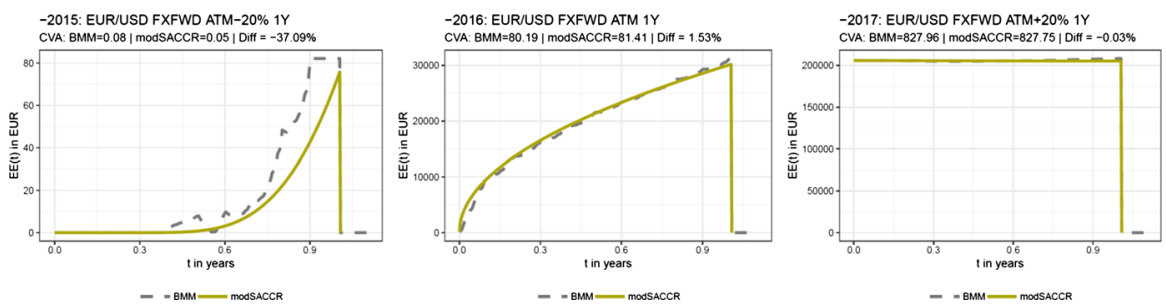

Fig. 9 Expected exposure of EUR/USD 1Y FX forwards. Note The figure shows the expected exposure profile and CVA results of EUR/USD 1Y FX forwards with different moneyness. The solid line represents the result from the modified SA-CCR, while the dashed line shows the result based on the benchmark model
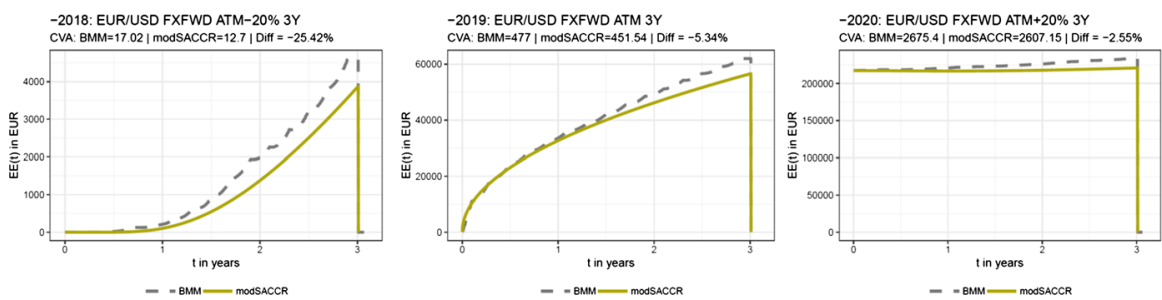

Fig. 10 Expected exposure of EUR/USD 3Y FX forwards. Note The figure shows the expected exposure profile and CVA results of EUR/USD 3 Y FX forwards with different moneyness. The solid line represents the result of the modified SA-CCR, while the dashed line shows the result based on the benchmark model
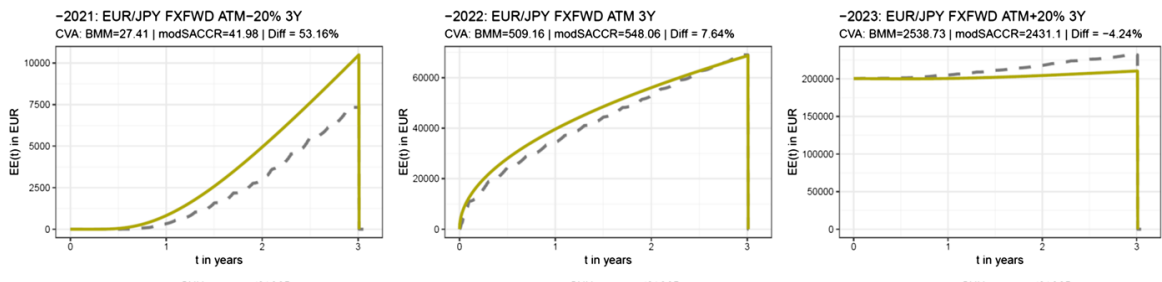

Fig. 11 Expected exposure of EUR/JPY 3Y FX forwards. Note The figure shows the expected exposure profile and CVA results of EUR/JPY 3Y FX forwards with different moneyness. The solid line represents the result of the modified SA-CCR, while the dashed line shows the result based on the benchmark model

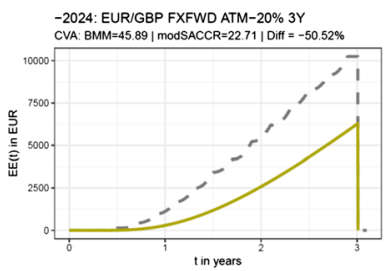

- - BMM- modSACCR

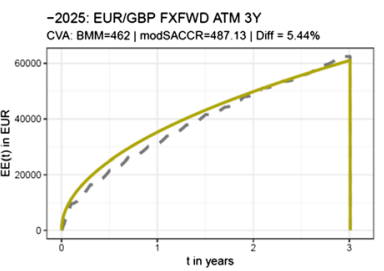

- - BMn — modsacCR

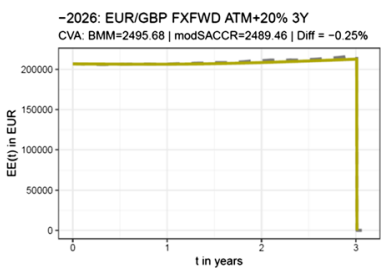

- - вм4 - modsaccr

Fig. 12 Expected exposure of EUR/GBP 3Y FX forwards. Note The figure shows the expected exposure profile and CVA results of EUR/GBP 3 Y FX forwards with different moneyness. The solid line represents the result of the modified SA-CCR, while the dashed line shows the result based on the benchmark model 

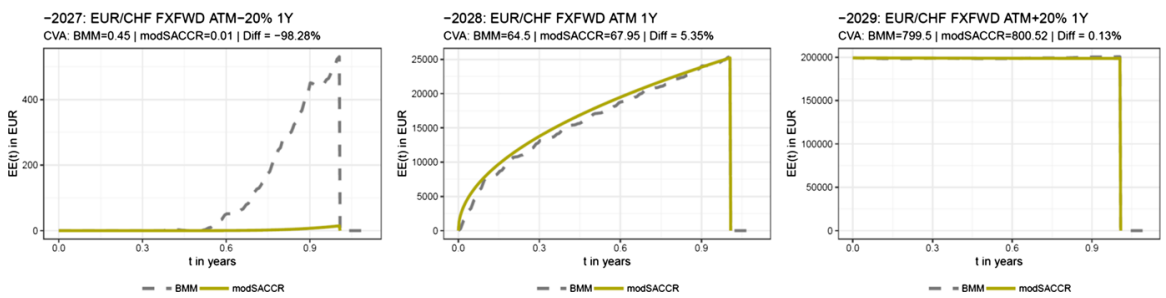

Fig. 13 Expected exposure of EUR/CHF 1Y FX forwards. Note The figure shows the expected exposure profile and CVA results of EUR/CHF 3Y FX forwards with different moneyness. The solid line represents the result of the modified SA-CCR, while the dashed line shows the result based on the benchmark model
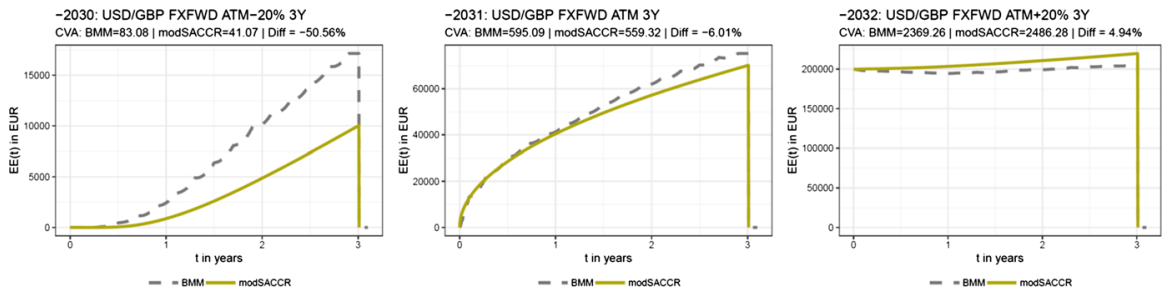

Fig. 14 Expected exposure of USD/GBP 3Y FX forwards. Note The figure shows the expected exposure profile and CVA results of USD/GBP 3Y FX forwards with different moneyness. The solid line represents the result of the modified SA-CCR, while the dashed line shows the result based on the benchmark model

\section{A.4.5 Output: profiles for combined netting sets}

See Figs. 15, 16, and 17.
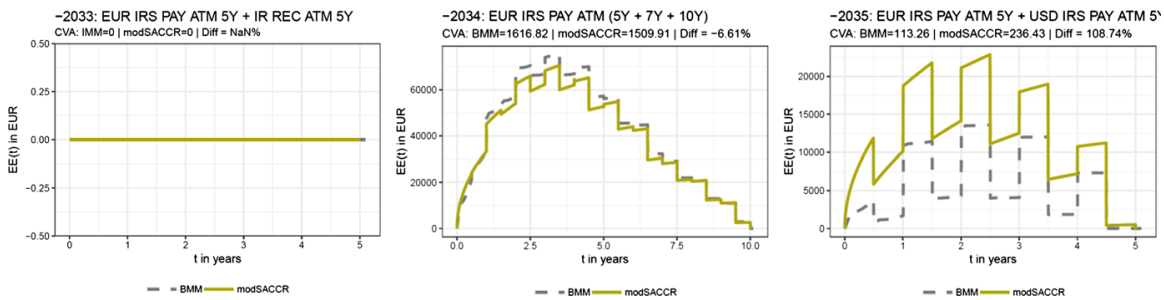

Fig. 15 Expected exposure profile of IR multi-transaction netting sets. Note This figure provides the expected exposure profile and CVA results for the hypothetical netting sets 2033, 2034 and 2035. The solid line represents the result of the modified SA-CCR, while the dashed line shows the result based on the benchmark model 


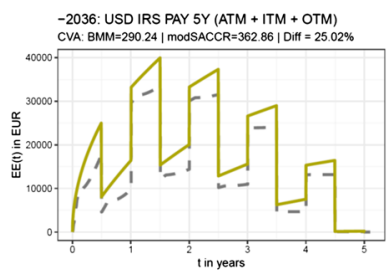

- - BMM - mossacCR

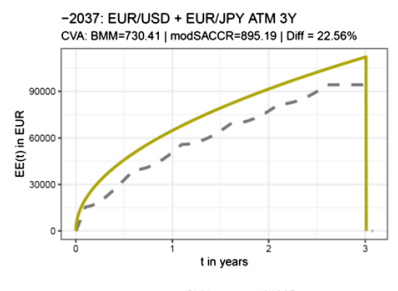

-- BnM- moSAACCR

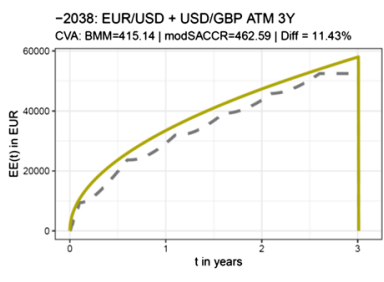

- - BMM - modSACCR

Fig. 16 Expected exposure profile of multi-transaction netting sets. Note This figure provides the expected exposure profile and CVA results for the hypothetical netting sets 2036, 2037 and 2038. The solid line represents the result of the modified SA-CCR, while the dashed line shows the result based on the benchmark model

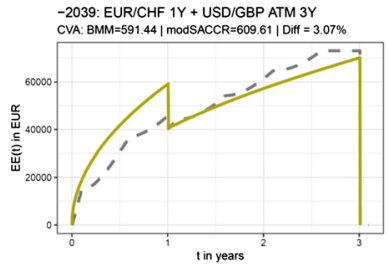

- - BMM- mOOSACCR

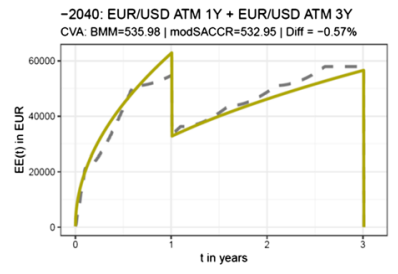

- - BMM- modSACCR

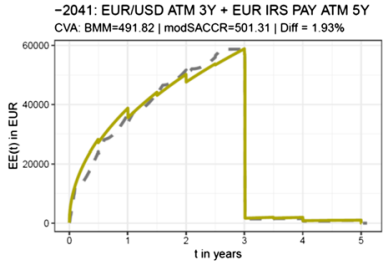

- BMM - modsaCCR

Fig. 17 Expected exposure profile of IR multi-transaction netting sets. Note This figure provides the expected exposure profile and CVA results for the hypothetical netting sets 2039, 2040 and 2041. The solid line represents the result of the modified SA-CCR, while the dashed line shows the result based on the benchmark model

\section{A.4.6 Output: profiles for portfolios with perfect CSA}

See Figs. 18 and 19.

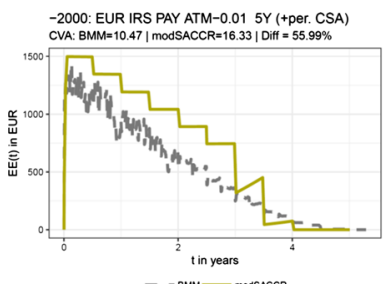

- - вмM- modsaccr

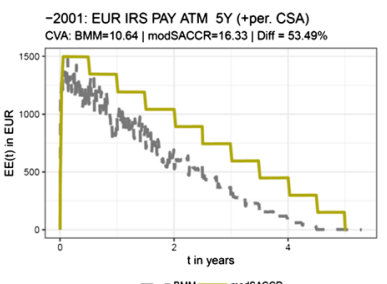

- - sum - mossacce

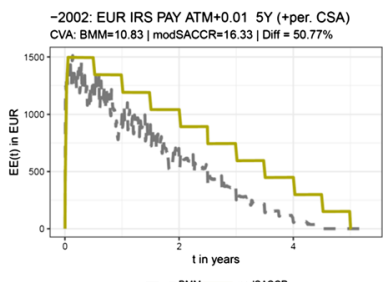

- - вмм - modsaccr

Fig. 18 Expected exposure profile of IR swaps with perfect CSA. Note This figure provides the expected exposure profile and CVA results for the hypothetical netting sets 2000, 2001 and 2002 under the assumption of a perfect CSA. The solid line represents the result of the modified SA-CCR, while the dashed line shows the result based on the benchmark model 

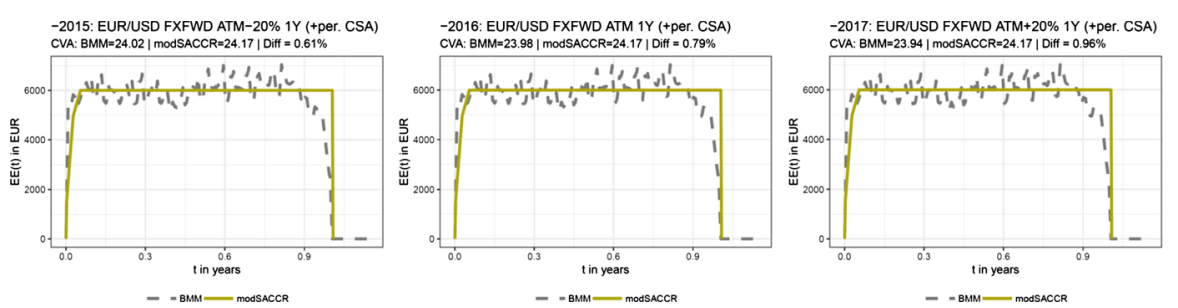

Fig. 19 Expected exposure profile of FX forwards with perfect CSA. Note This figure provides the expected exposure profile and CVA results for the hypothetical netting sets 2015, 2016 and 2017 under the assumption of a perfect CSA. The solid line represents the result of the modified SA-CCR, while the dashed line shows the result based on the benchmark model

\section{A.4.7 Output: profiles for portfolios with imperfect CSA}

See Figs. 20, 21.
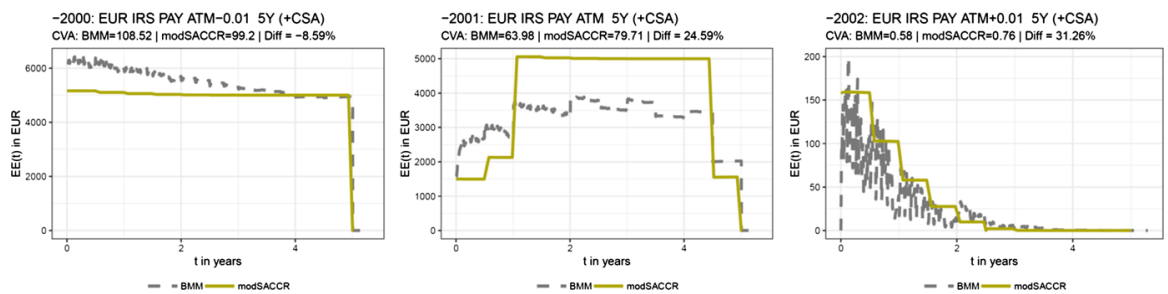

Fig. 20 Expected exposure profile of IR swaps with a CSA $(\mathrm{TH}=5.000, \mathrm{MTA}=1.000)$. Note This figure provides the expected exposure profile and CVA results for the hypothetical netting sets 2000, 2001 and 2002 under the assumption of an imperfect CSA. The solid line represents the result of the modified SA$\mathrm{CCR}$, while the dashed line shows the result based on the benchmark model
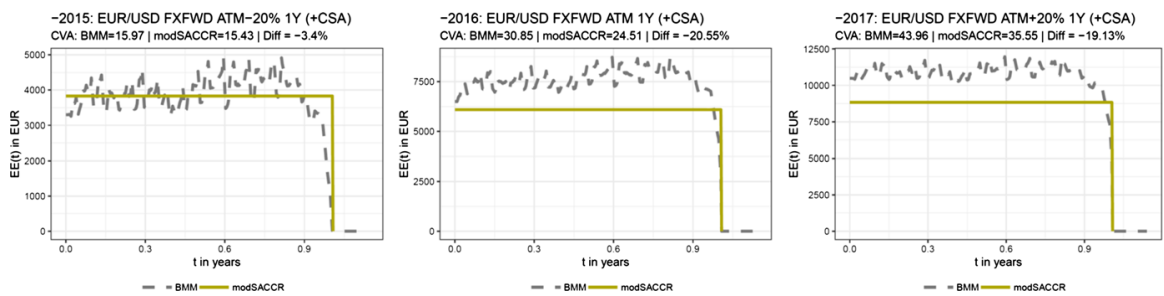

Fig. 21 Expected exposure profile of FX forwards with a CSA (TH=5.000,MTA=1.000). Note This figure provides the expected exposure profile and CVA results for the hypothetical netting sets 2000, 2001 and 2002 under the assumption of an imperfect CSA. The solid line represents the result of the modified SA-CCR, while the dashed line shows the result based on the benchmark model 


\section{A.4.8 Output: profiles for FX Options}

See Figs. 22 and 23.

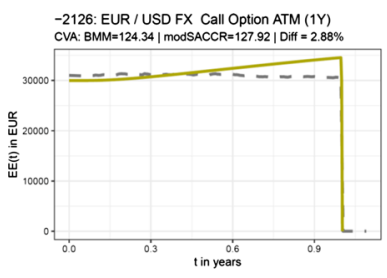

- BMm - modsacca

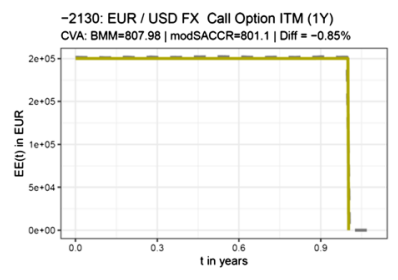

- - вмм - modsaccr

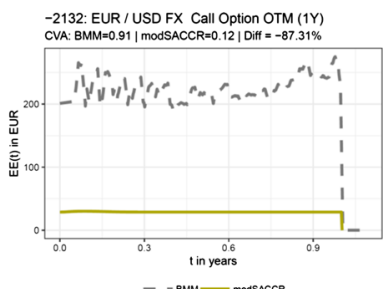

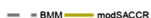

Fig. 22 Expected exposure profile of FX options. Note This figure provides the expected exposure profile and CVA results for the hypothetical netting sets 2126, 2130 and 2132. The solid line represents the result of the modified SA-CCR, while the dashed line shows the result based on the benchmark model

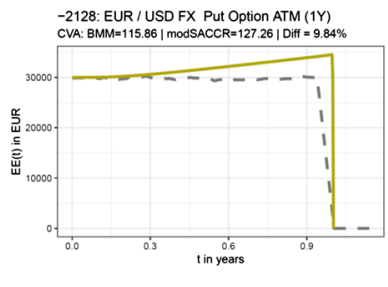

- - вим - mosasacce

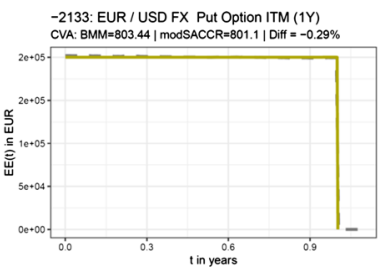

- - sum - mossaccr

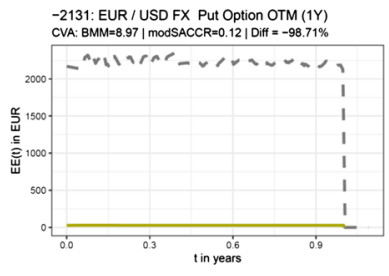

- - вмm - modsacce

Fig. 23 Expected exposure profile of FX options. Note This figure provides the expected exposure profile and CVA results for the hypothetical netting sets 2128,2131 and 2133. The solid line represents the result of the modified SA-CCR, while the dashed line shows the result based on the benchmark model 


\section{References}

BCBS. (1996). Amendment to the capital accord to incorporate market risks. Basel Committee on Banking Supervision Updated version (November 2005).

BCBS. (2005). The application of Basel II to trading activities and the treatment of double default effects. Basel Committee on Banking Supervision.

BCBS. (2013). Consultative document - the non-internal model method for capitalising counterparty credit risk exposures. Basel Committee on Banking Supervision.

BCBS. (2014a). Basel III leverage ratio framework and disclosure requirements. Basel Committee on Banking Supervision.

BCBS. (2014b). Foundations of the standardised approach for measuring counterparty credit risk exposures. In Working paper no 26. Basel Committee on Banking Supervision.

BCBS. (2014c). The standardised approach for measuring counterparty credit risk exposures. Basel Committee on Banking Supervision.

BCBS. (2014d). Supervisory framework for measuring and controlling large exposures. Basel Committee on Banking Supervision https://www.bis.org/publ/bcbs283.pdf

BCBS. (2017). Basel III: Finalising post-crisis reforms. Basel Committee on Banking Supervision https:// www.bis.org/bcbs/publ/d424.pdf

BCBS. (2019). Minimum capital requirements for market risk (rev. version). Basel Committee on Banking Supervision.

BCBS, IOSCO. (2015). Margin requirements for non-centrally cleared derivatives. Basel Committee on Banking Supervision.

Brigo, D., \& Masetti, M. (2005). A formula for interest rate swaps valuation under counterparty risk in presence of netting agreements. http://www.damianobrigo.it

Canabarro, E., \& Duffie, D. (2003). Measuring and marking counterparty credit risk. Institutional Investor Books, chap, 9, pp. 122-134.

Cherubini, U. (2013). Credit valuation adjustment and wrong way risk. Quantitative Finance Letters, 1(1), $9-15$.

EBA. (2016). Response to the European commisssion's CFA on standard approach for counterparty credit risk and own funds requirements for market risk. European Banking Authority.

EBA. (2017). Discussion paper - implementation in the European union of the revised market risk and counterparty credit risk frameworks. European Banking Authority.

ESAs. (2016). Final draft regulatory technical standards on risk-mitigation techniques for OTC-derivative contracts not cleared by a CCP under article 11(15) of regulation (eu) no. 648/2012. Risk-management procedures for non-centrally cleared OTC derivatives.

Fleck, M., \& Schmidt, A. (2005). Counterparty credit risk modelling: Risk management pricing and regulation. Risk Books, chap Analysis of Basel II treatment of counterparty risk, pp. 229-261.

Gregory, J. (2010). Counterparty credit risk and credit value adjustments (2nd ed.). London: Wiley.

Gregory, J. (2015). The XVA challenge: Counterparty credit risk, funding, collateral and capital (3rd ed.). London: Wiley.

Heston, S. L. (1993). A closed solution for options with stochastic volatility, with application to bond and currency options. Review of Financial Studies, 6(2), 327-343.

Hull, J., \& White, A. (2012). CVA and wrong way risk. Financial Analysts Journal, 68(5), 58-69.

ISDA, TBMA, LIBA. (2003). Counterparty risk treatment of OTC derivatives and securities financing transactions. ISDA https://www.sec.gov/rules/proposed/s72103/isda020404a.pdf.

Kao, L. J. (2016). Credit valuation adjustment of cap and floor with counterparty risk: A structural pricing model for vulnerable European options. Review of Derivatives Research. https://doi.org/10.1007/ s11147-015-9114-7.

Leung, S. Y., \& Kwok, Y. K. (2005). Credit default swap valuation with counterparty. The Kyoto Economic Review, 74(1), 25-45.

Lynch, D. (2014). Counterparty risk management: Measurement, pricing and regulation, risk books, chap the Basel III enhancements to counterparty risk capital charges, pp. 3-16.

Marquart, M. (2016). Cva for pricing: Comparison of CEM, SA-CCR, and advanced approach. Wilmott, 2016(86), 52-59. https://doi.org/10.1002/wilm.10550.

Picoult, E. (2004). Economic capital for counterparty credit risk. The RMA Journal, 86(6), 44-51. 
Pricso, B. D., Rosen, D. (2005). Counterparty credit risk modelling: Risk management, pricing and regulation. Risk Books, chap Modelling stochastic counterparty credit exposures for derivatives portfolios, pp. 3-48.

Pykhtin, M. (2014) Counterparty risk management: Measurement, pricing and regulation, risk books, chap the non-internal model method for counterparty credit risk, pp. 33-57.

Pykhtin, M., \& Zhu, S. (2007). A guide to modeling counterparty credit risk. GARP Risk Review 37.

Sorensen, E. H., \& Bollier, T. F. (1994). Pricing swap default risk. Financial Analysts Journal, 50(3), $23-33$.

Thompson, T., \& Dahinden, V. (2013). Counterparty risk and CVA survey: Current market practice around counterparty risk regulation, CVA management and funding. Deloitte and Solum Financial Partners.

Trolle, A. B., \& Schwartz, E. S. (2009). A general stochastic volatility model for the pricing of interest rate derivatives. The Review of Financial Studies, 22(5), 2002-2057.

Publisher's Note Springer Nature remains neutral with regard to jurisdictional claims in published maps and institutional affiliations. 